
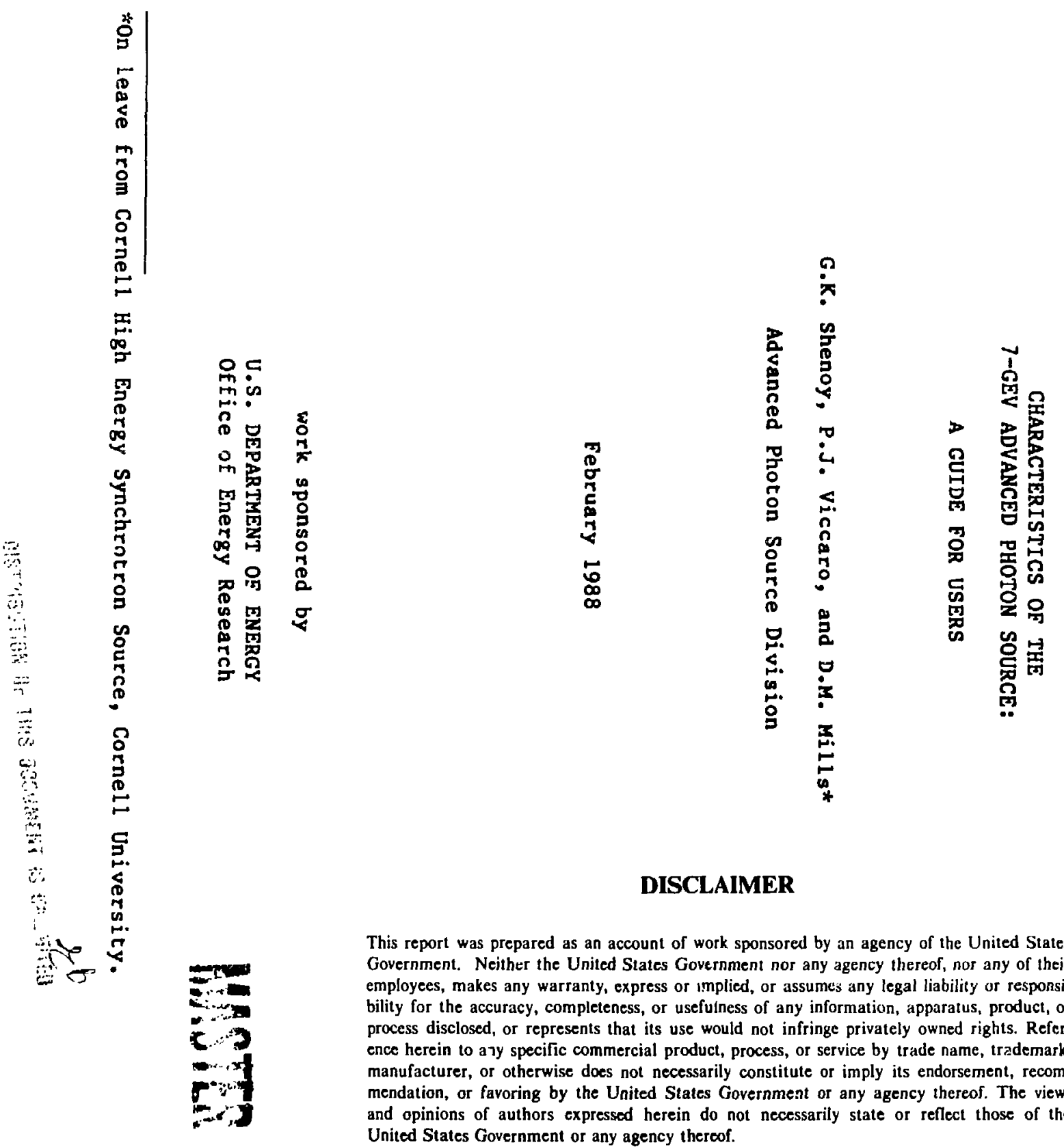

\title{
DISCLAIMER
}

This report was prepared as an account of work sponsored by an agency of the United States Government. Neither the United States Government nor any agency thereof, nor any of their employees, makes any warranty, express or implied, or assumes any legal liability or responsibility for the accuracy, completeness, or usefuiness of any information, apparatus, product, or process disclosed, or represents that its use would not infringe privately owned rights. Reference herein to a yy specific commercial product, process, or service by trade name, trademark, manufacturer, or otherwise does not necessarily constitute or imply its endorsement, recommendation, or favoring by the United States Government or any agency thereof. The views and opinions of authors expressed herein do not necessarily state or reflect those of the United States Government or any agency theroof. 
ACKNOWLEDGMENTS....................................vi

ABSTRACT $\quad \ldots \ldots \ldots \ldots \ldots \ldots \ldots \ldots \ldots \ldots \ldots \ldots \ldots \ldots \ldots \ldots \ldots \ldots \ldots \ldots \ldots \ldots \ldots \ldots$

1 PURPOSE.........................................

2 PARAMETERS OF THE 7-GEV ADVANCED PHOTON SOURCE...............

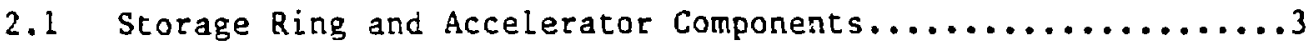

2.2 Sector Layout..................................

3 CHOICE OF STORAGE-RING ENERGY AND UNDULATOR TUNABILITY.........

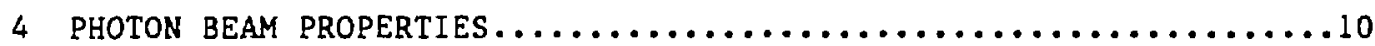

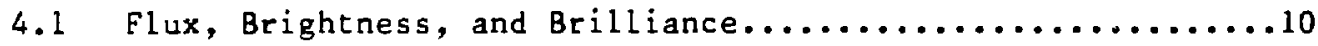

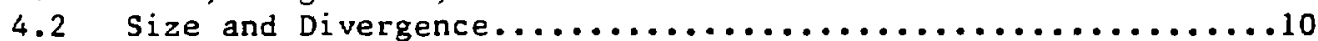

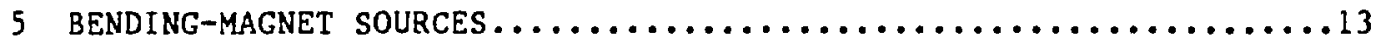

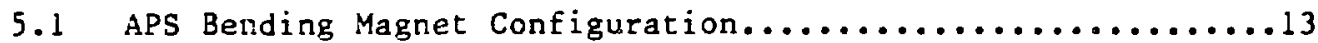

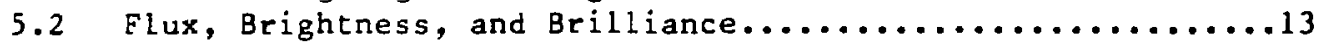

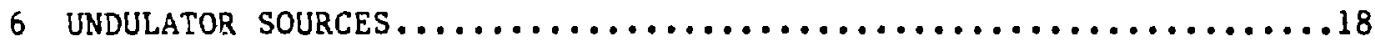

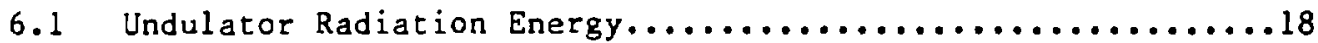

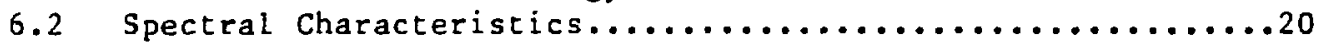

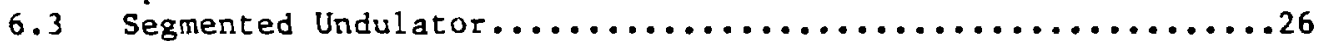

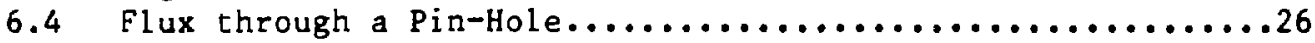

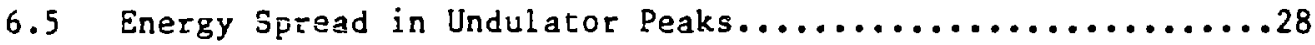

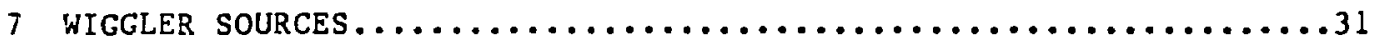

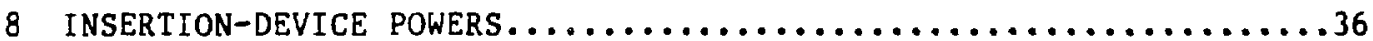

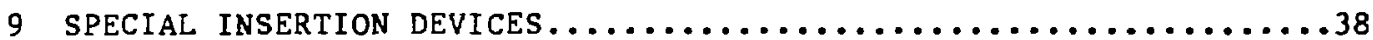

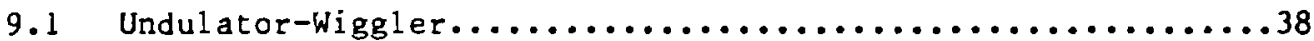

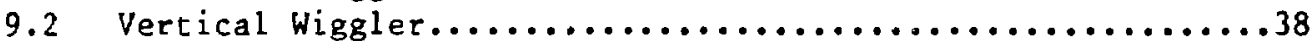

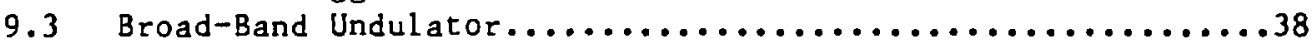

9.4 Extra-Long Straight Sections.....................40

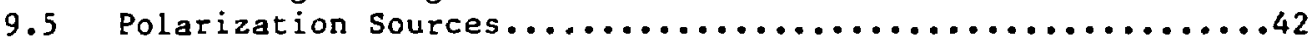

10 TIME STRUCTURE.......................................

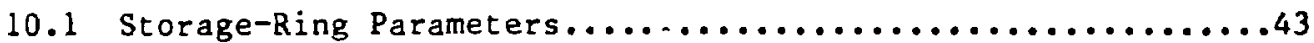

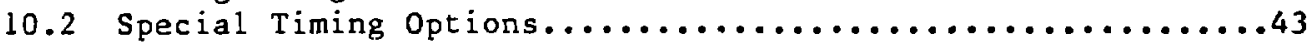

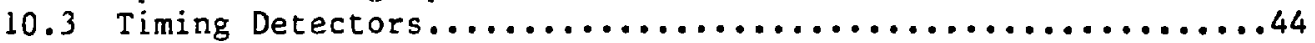

10.4 Flux per Bunch for Various APS Sources................. 45

11 EXPERIMENTAL FLOOR AND LABORATORY OFEICE MODULES .............46

11.1 General Layout...............................46

11.2 Laboratory/Office Modules for Users..................46

REFERENCES...................................... 
FIGURES

2.1 Layout of the $7-\mathrm{GeV}$ Advanced Photon Source.................

2.2 Layout of Magnets in One of the 40 Sectors of the APS Storage Ring....................................

3.1 Storage-Ring Energy Necessary for a Nd-Fe-B Undulator to Achieve the First-Harmonic Tunability from about 4.7 to $14 \mathrm{keV}$, as a Function of Minimum Insertion-Device Gap................

5.1 Flux from a Bending Magnet on Various Synchrotron Sources......14

5.2 Brightness of Bending-Magnet Radiation from Various

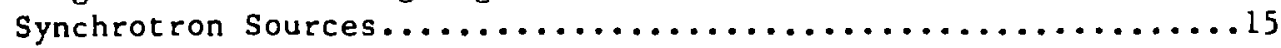

5.3 Brilliance of Bending-Magnet Radiation from Various Synchrotron Sources................................

6.1 On-Axis Brilliance vs. Energy for APS Undulator A at $100 \mathrm{~mA}$,

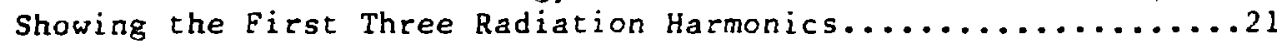

6.2 On-Axis Brilliance of the First-Harmonic Radiation from APS

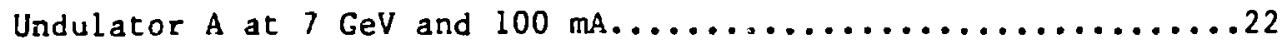

6.3 On-Axis Brilliance of the First-Harmonic Radiation from APS

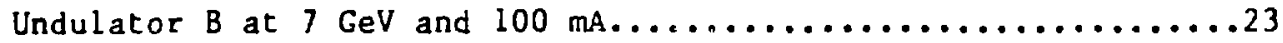

6.4 On-Axis Brilliance from APS Undulator $C\left(\lambda_{0}=20 \mathrm{~cm}\right)$ at

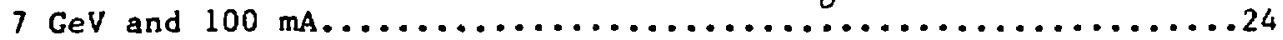

6.5 Angular Distribution of the Eirst-Harmonic Radiation from APS Undulator A along the $x-$ and $y$-Axes, Showing the $y-$

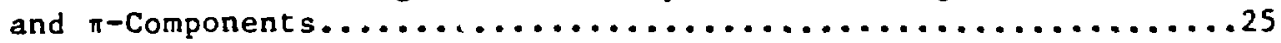

6.6 Flux through a Set of Pin-Holes Showing the First-Harmonic Energy Spectra from APS Undulator $\mathrm{A}$ at $7 \mathrm{GeV}$ and $100 \mathrm{~mA} \ldots \ldots . .27$

6.7 On-Axis Brillance, Brightness, and Flux through a Pin-Hole of Size 22 urad $\times 22$ rad Placed along the Axis of Undulator A vs. X-Ray Energy of the First Harmonic...........29

7.1 Flux from Wigglers on the 7-GeV APS at $100 \mathrm{~mA}$, Compared with Flux from Wigglers on Other Synchrotron Sources.............33

7.2 Central Brightness from Wigglers on the $7-\mathrm{GeV}$ APS at $100 \mathrm{~mA}$, Compared with That from Wigglers on Other Synchrotron Sources...34

7.3 Central Brilliance from Wigglers on the 7-GeV APS at $100 \mathrm{~mA}$, Compared with That from Wigglers on Other Synchrotron Sources...35

9.1 On-Axis Spectral Brilliance vs. Photon Energy for the Undulator-

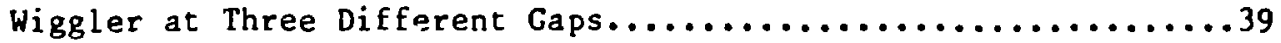




\section{FIGURES (Cont'd)}

9.2 On-Axis Brilliance of a Broad-Band Undulator, Showing the Subspectra of Each of the Segments.....................41

11.1 Layout of Typical Beam Lines in a Part of the Storage-Ring Experimental Hall................................47

11.2 Typical Laboratory/Office Module Providing Support for Four Insertion Devices and Four Bending-Magnet Beam Lines..........48

\section{TABLES}

2.1 Design Parameters for the APS Storage Ring................6

4.1 Values of B-Eunctions in Different Parts of the Lattice and Dimensions of Different Sources.....................11

5.1 Parameters of Various Bending-Magnet Sources...............17

6.1 Characteristics of Three Selected Undulators on the 7-GeV APS...19

7.1 Parameters for Various Wiggler Sources....................32

8.1 Power Distributions from Insertion Devices....................

10.1 Time Structures of Various Storage Rings.................44

10.2 Flux per Bunch for APS $x$-Ray Sources...................45 


\section{ACKNOWLEDGMENTS}

The authors wish to thank all the members of the APS Division for their support in the preparation of this document. We especially appreciate the effort of Bonnie Meyer in the typing of the manuscript and the effort of Floyd Bennett in editing the document. 


\title{
CHARACTERISTICS OF THE \\ 7-GEV ADVANCED PHOTON SOURCE:
}

\author{
A GUIDE FOR USERS
}

\author{
G.K. Shenoy, P.J. Viccaro, and D.M. Mills
}

\begin{abstract}
In this document we present the characteristics of the electromagnetic radiation from various types of sources on the 7-GeV Advanced Photon Source (APS) storage ring. The sources include bending magnets, undulators, and wigglers. The characteristics are compared with those of other synchrocron sources when operated at their design specifications. The influence of positron beam size on the on-axis brilliance is discussed, along with the power distribution from these sources. The goal of this document is to provide users with enough information on the characteristics of radiation from the APS storage ring so that experiments can be efficiently planned.
\end{abstract}

\section{PURPOSE}

During the past two years, the design work on the 7-GeV Advanced Photon Source (APS) has progressed: the construction of this national user facility can begin in 1989. Towards this accomplishment, many accelerator physicists, engineers, architects, and users have contributed. In addition, the APS project staff have had the benefit of many advisory committees and working groups, with memberships from within and outside of the Laboratory, sharing this major design task. We wish specifically to mention the National Task Group, whose efforts led to an upgrade of the storage-ring energy from $6 \mathrm{GeV}$ to $7 \mathrm{GeV}$, and the Conventional Facilities Subcommittee (appointed by the APS User Organization Steering Committee), which assisted in enhancing the experimental facility. The efforts of both these groups have greatly increased the potential use of the APS facility.

Many workshops have been held on the design of the accelerator and its components. 2,3 The designs of the accelerator components and the storage ring have been subjected to detcailed reviews by the U.S. Department of Energy (DOE) office of Energy Research ${ }^{4}$ and by the Accelerator Advisory Committee appointed by the APS. The parameters of the design are now nearly finalized.

In addition, many workshops have been held to asses the implications of the Advanced Photon Source for various user communities. 5 The First Users Meeting for the Advanced Photon Source was held November 14-15, 1986, at Argonne. 
The present design of the facility reflects the close interaction between the users and the staff of the Advanced Photon Source. Over the next few years, the users will design beam lines and experimental facilities needed to conduct their research at this facility. To facilitate this effort and to enhance the ongoing user interaction with the APS staff, we have documented the radiation characteristics of the APS and the layout of the experimental hall in the current design. This report contains an $1 p^{-t}, p^{-d a t e}$, expanded version of information provided in earlier documents. 10,11

In designing the next generation of experiments at the APS, the past experience of users at operating synchrotron facilities will serve as an index of comparison with the expected performance of the APS. In many places we have 1 isted the radiation characteristics of sources on existing storage rings and compared these characteristics with those of the APS. In calculating these radiation characteristics, of course, we have used information current at the time this document was prepared. The calculated parameters may not correspond exactly to the final operating parameters of the APS.

References pertinent to the present effort are cited throughout this report. However, no attempt has been made to provide an exhaustive bibliography of synchrotron radiation sources or their design characteristics. 


\section{PaRAMETERS OF THE 7-GEV ADVANCED PHOTON SOURCE}

\subsection{STORAGE RING AND ACCELERATOR COMPONENTS}

The general layout of the storage ring is shown in Fig. 2.1.12 The linac ( 1 inear accelerator) provides three-ampere (3-A) electron pulses at $200 \mathrm{MeV}$. These electrons bombard a tungsten target to produce positrons, which are focused and accelerated further to $450 \mathrm{MeV}$. The positrons are stored in a positron accumulator ring (PAR) located near the end of the linac building before being injected into a booster synchrotron. In the booster synchrotron the positrons are accelerated in two stages, first to $2.73 \mathrm{GeV}$ and then to 7.0 $\mathrm{GeV}$ (or to $7.7 \mathrm{GeV}$, if necessary). The $7-\mathrm{GeV}$ positron bunches are then extracted and injected into the storage ring at energy. In all, $2.2 \times 10^{12}$ positrons are needed to generate a 100-mA current. The estimated injection time from a cold start is about four minutes.

The electron/positron linac has a total length of about $40 \mathrm{~m}$. The PAR has a circumference of $9 \mathrm{~m}$. The positron trajectory in the booster synchrotron is about $367 \mathrm{~m}$. This ring, with $40 \mathrm{cell} \mathrm{s}$, has 68 bending magnets and many focusing elements. The $7-\mathrm{GeV}$ positron beam will achieve a natural emittance of $132 \mathrm{~nm}$-rad before injection into the storage ring.

\subsection{SECTOR LAYOUT}

The emittance of a positron beam in a storage ring is proportional to the square of the beam energy and inversely proportional to the third power of the number of sectors. In the case of the $7-\mathrm{GeV}$ APS, the low value of the natural emittance ( $8 \mathrm{~nm}-\mathrm{rad}$ ) is achievable by having 40 sectcrs in the storage ring; this also allows one to have 40 straight sections. Figure 2.2 shows a layout of the magnetic components in a sector of this Chasman-Green lattice, along with the insertion-device and bending-magnet beam lines. Each sector contains an achromatic bending system made up of two dipole magnets, each providing a $4.5^{\circ}$ bend. Each sector also has a dispersion-free region of $6.2 \mathrm{~m}$ length that can accommodate 5-m-long insertion devices. Thus, the storage ring contains 40 straight sections, 80 bending magnets, 400 quadrupoles, and 280 sextupoles. There are also 678 dipole correctors. Al1 the magnetic components are optimized for 7-GeV operation and capable of $7.7-\mathrm{GeV}$ operation. The placement of all these components requires a storage-ring circumference of $1060 \mathrm{~m}$.

It is estimated that the positron beam 1 ifetime will exceed $10 \mathrm{~h}$ with a beam-on operating pressure of 1 nTorr in the storage ring. This pressure will be achieved by the use of distributed pumps made up of Nonevaporable Getter (NeG) strips and lumped ion pumps.

The $\mathrm{rf}$ (radio frequency) system operates at $353 \mathrm{MHz}$ and can deliver 1.27 MW of power to operate the ring at $7 \mathrm{GeV}$ and $100 \mathrm{~mA}$. The total installed rf power will be $3.0 \mathrm{MW}$. There are 1248 sites ( $r f$ buckets) for the positron bunches, but a 100-mA beam can be stored by filling only about 20 of these buckets.

The APS design includes diagnostic instrumentation to monitor the positron beam position, beam profile, and current and beam losses. There will 


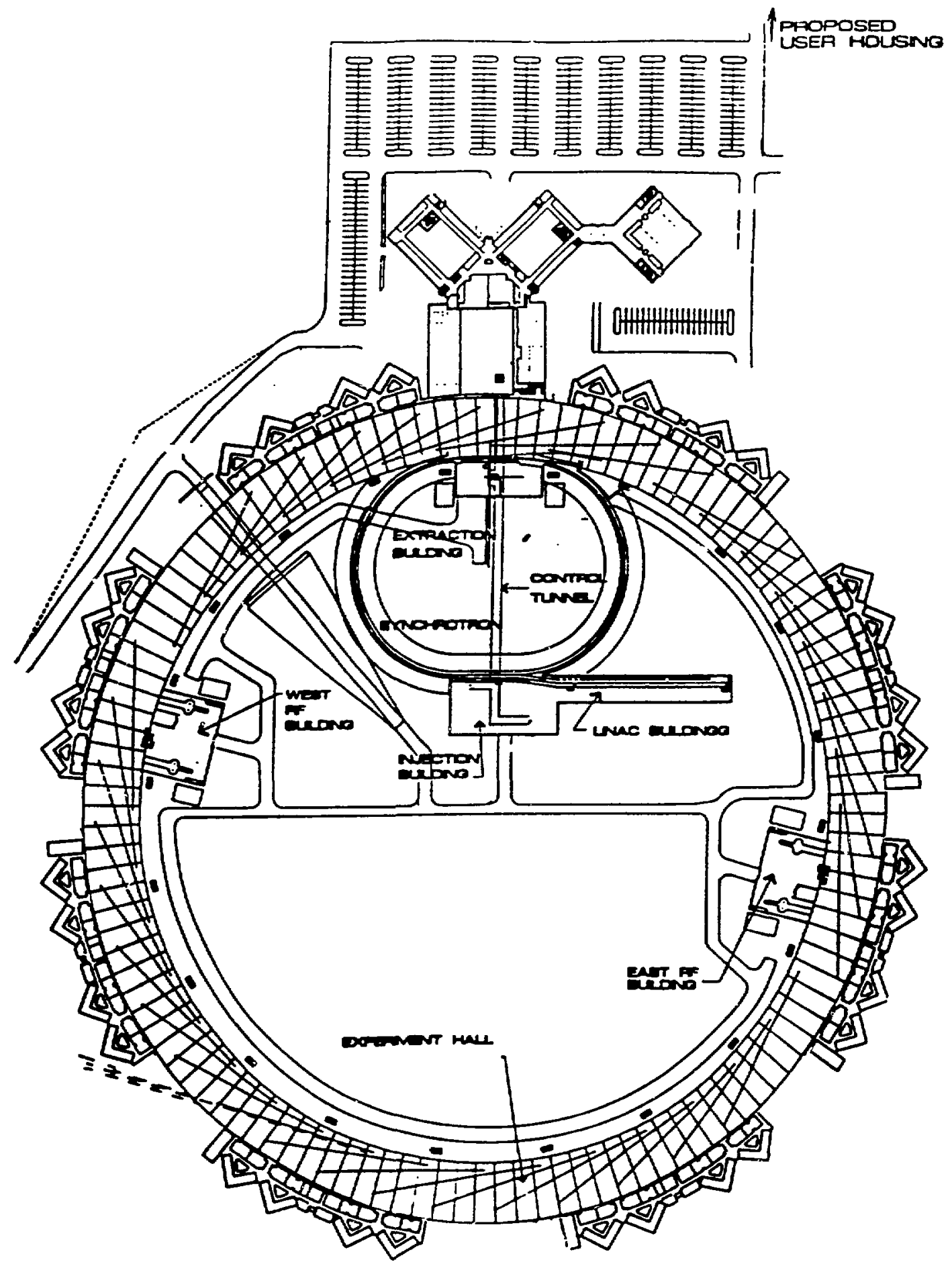

FIGURE 2.1 Layout of the 7-GeV Advanced Photon Source (The positron accumulator ring is located near the end of the linac building.) 


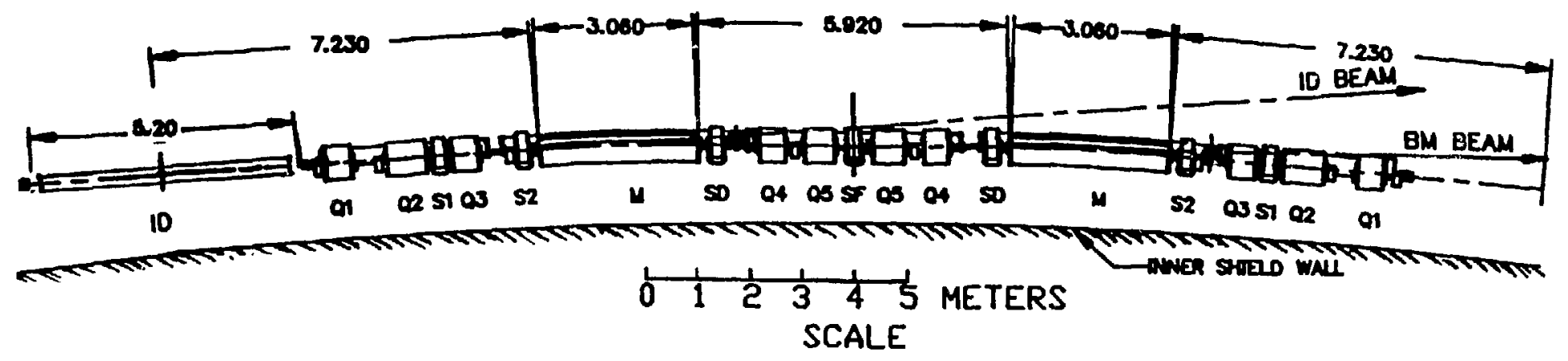

FICURE 2.2 Layout of Magnets in One of the 40 Sectors of the APS Storage Ring 
be 360 positron beam monitors, 80 loss monitors, and 40 fluorescent-screen profile monitors. In addition, the photon beam from each of the insertion devices will be monitored by horizontal and vertical beam position detectors incorporated in a feedback loop to stabilize the phase space of the posit:on beam.

Of the 40 straight sections, 34 will be available for the insertion devices. The remaining six sections are reserved for the storage-ring accelerator hardware. Of these, one is used for injection, a second for beam abort, three for $r f$ cavities, and one for positron beam diagnostics. Although the ring incorporates 80 bending magnets, only 40 of them can be equipped with beam lines (see Fig. 2.2). The accelerator hardware shadows five of these 40 bending magnets, so that the maximum number of bending-magnet sources is 35 on this lattice.

Table 2.1 provides a complete 1 ist of storage-ring paraneters.

TABLE 2.1 Design Parameters for the APS Storage Ring

\begin{tabular}{|c|c|}
\hline Parameter & Value \\
\hline $\begin{array}{l}\text { Positron Energy (GeV) } \\
\text { Injection Energy (GeV) } \\
\text { Beam Current (mA) } \\
\text { Beam Lifetime (h) }\end{array}$ & $\begin{array}{l}7(+10 \%) \\
7(+10 \%) \\
100 \\
10\end{array}$ \\
\hline $\begin{array}{l}\text { Horizontal Emittance (nm-rad) } \\
\text { Vertical Emittance (nm-rad) }\end{array}$ & $\begin{array}{l}7 \\
0.7\end{array}$ \\
\hline $\begin{array}{l}\text { Straight Sections } \\
\text { Insertion Devices (IDs) } \\
\text { Standard Length of IDs (m) } \\
\text { Single Undulator Tunability (keV) } \\
\text { First-Harmonic Radiation } \\
\text { Third-Harmonic Radiation }\end{array}$ & $\begin{array}{l}40 \\
34 \\
5 \\
4.7 \text { to } 14 \\
14 \text { to } 42\end{array}$ \\
\hline $\begin{array}{l}\text { Number of Bending Magnets } \\
\text { Bending-Magnet Sources } \\
\text { Length of Bending Magnet (m) } \\
\text { Bend Radius (m) } \\
\text { Critical Energy (keV) }\end{array}$ & $\begin{array}{l}80 \\
35 \\
3.06 \\
38.96 \\
19.5\end{array}$ \\
\hline $\begin{array}{l}\text { Radio Frequency (MHz) } \\
\text { Storage-Ring Circumference (m) } \\
\text { Revolution Time ( } \mu s) \\
\text { Bunch Width (ps) } \\
\text { Maximum rf Buckets }\end{array}$ & $\begin{array}{l}352.96 \\
1060 \\
3.5358 \\
119 \\
1248\end{array}$ \\
\hline $\begin{array}{l}\text { Typical Number of Bunches } \\
\text { Maximum Single-Bunch Current (mA) }\end{array}$ & 1 to 60 \\
\hline
\end{tabular}




\section{CHOICE OF STORAGE-RING ENERGY AND UNDULATOR TUNABILITY}

The energy of the positrons for the APS storage ring was chosen to be $6 \mathrm{GeV}$ at an early stage in its design. This choice would satisfy the original user specification of obtaining $20-\mathrm{keV}$ radiation from the first harmonic of an undulator. ${ }^{3}$ However, as additional capabilities of undulator sources were investigated, it became evident that at $6 \mathrm{GeV}$ the energy tunability of radiation from undulators is rather limited for first-harmonic energies above 10 $\mathrm{keV}$. As a result, as many as five andulators would be needed at every straight section of the storage ring to span the photon energy interval of 4 to $20 \mathrm{keV}$. Each of the five undulators would cover a small energy range. 10,11 Hence, in an appendix to the $6-\mathrm{GeV}$ design document, 13 we suggested the need to increase the storage-ring energy, possibly to $7 \mathrm{GeV}$.

A National Task Group was formed to address the question of ring energy selection for the APS and its effects on undulator tunability. 1 This group set certain tunability criteria for the APS undulators by incorporating the information on user requirements presented in a large number of documents and articles - - in particular, the Scientific Case Workshop Report. 5 The criteria also reflected optimum utilization of the facility during its initial and mature stages of operation. During the initial stage, indulator vacuum chambers with a large aperture $(12 \mathrm{~mm})$ for the positron beam may be necessary for operation of the ring. During the mature stage of operation, smaller apertures $(-8 \mathrm{~mm}$ ) would be possible. Both apertures set lower limits on the closed-gap value of the undulators, which in turn is one of the major factors determining the tunability range of the undulator.

The operational criteria recommended by the National Task Group are as follows:

- For the initial phase of operation, an undulator should provide first-harmonic radiation tunable over the range of 7 to $14 \mathrm{keV}$. In addition, $20-\mathrm{keV}$ radiation should be available in the first harmonic of a second undulator with a different period, or, if possible, in the third harmonic of the first undulator.

- In the mature phase of operation, the minimum gap and the ring energy should allow for the first undulator to be tunable over an interval of 4.7 to $14 \mathrm{keV}$. A second undulator should deliver $20-\mathrm{keV}$ radiation. Again, although insertion of two devices per straight section is considered reasonable, the third-harmonic intensities of the first undulator might be large enough in many applications to dispense with having a second undulator on the same straight section.

The above criteria for the $\mathrm{Nd}-\mathrm{Fe}-\mathrm{B}$ hybrid undulators can be translated into relationships between gap and storage-ring energy. This is shown in Eig. 3.1. It can be seen that designing the storage ring to operate at 7.0 $\mathrm{GeV}$, with the capability to achieve energies up to $7.7 \mathrm{GeV}$, will permit us to meet the above criteria. This leads to the following operational plan: 


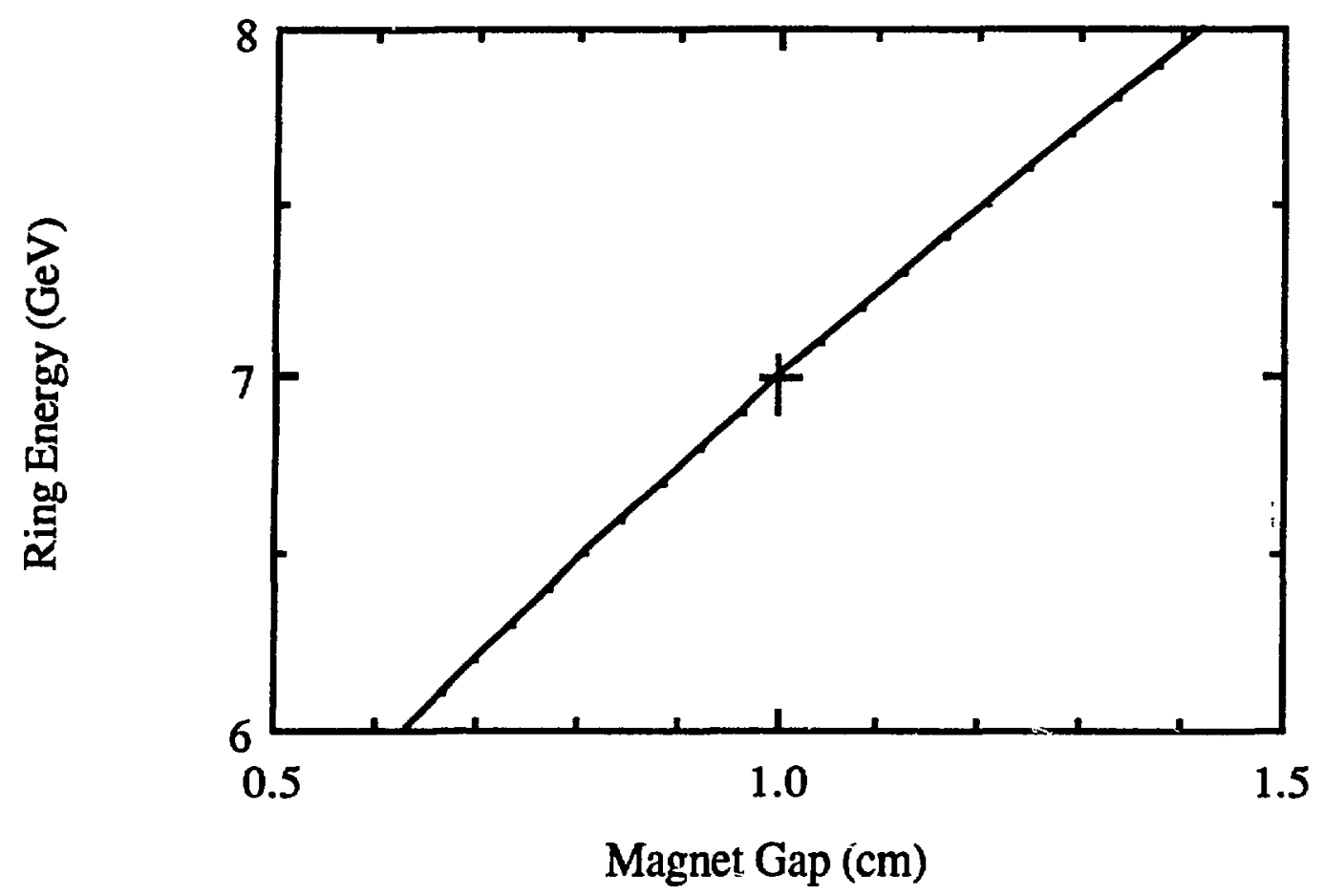

FIGURE 3.1 Storage-Ring Energy Necessary for a Nd-Fe-B Undulator to Achieve First-Harmonic Tunability from about 4.7 to $14 \mathrm{keV}$, as a Function of Minimum Insertion-Device Gap 
- During the initial period of operation, the insertiondevice vacuum chamber will permit a minimum gap of $1.4 \mathrm{~cm}$.

- The minimum gap will be reduced to $1.0 \mathrm{~cm}$ after mature operation is attained. This reduction will increase both the tunability and the photon brilliance.

- The undulators designed for the initial operation period will deliver their full performance potential during mature operation.

An undulator with a period of $3.3 \mathrm{~cm}$ fulfills all the operational criteria listed above. Details concerning the characteristics of such an undulator are presented in Sec. 6. 


\section{PHOTON BEAM PROPERTIES}

\subsection{FLUX, BRIGHTNESS, AND BRILLIANCE}

Three quantities can be used to characterize the radiated photons from the various sources on the APS: photon flux, spectral brightness, and spectral brilliance. The photon flux represents the number of photons per second with 0.17 energy bandwidth collected in unit angular spread $\theta$ in the positron orbit plane ( $x-z$ plane) and integrated over the entire vertical opening angle $\psi(y-z$ plane $)$. The spectral brightness defines the angular density of the photon flux, and the spectral brilliance is the photon intensity emitted in the unit phase-space volume of tra radiation field. The phase-space volume is obtained by convoluting the Gaussian distribution describing the positron beam in the storage ring and the radiation field, described in terms of Gaussian optics. Some authors prefer to use the term "brightness" for what we have defined as brilliance. Confusion can be avoided by bearing in mind the following units used for the quantities:

$$
\begin{aligned}
& \text { photon } \mathrm{flux}=\text { number of photons } / \mathrm{s} \cdot 0.1 \% \mathrm{BW} \cdot \mathrm{mrad} \theta \\
& \text { brightness }=\text { number of photons } / \mathrm{s} \cdot 0.1 \% \mathrm{BW} \cdot \mathrm{mrad} \theta \cdot \mathrm{mrad} \psi \\
& \text { brilliance }=\text { number of photons } / \mathrm{s} \cdot 0.1 \% \mathrm{BW} \cdot \mathrm{mrad}^{2} \cdot \mathrm{mm}^{2}
\end{aligned}
$$

\subsection{SIZE AND DIVERGENCE}

If $\Sigma_{i}$ and $\Sigma_{i}^{\prime}(i=x, y)$ are beam size and angular divergence, the on-axis (central) brilliance is obtained by dividing the on-axis (central) brightness by $2: \Sigma x^{\Sigma} y$. The "average value" of the on-axis spectral brilliance is obtained by dividing the photon flux by $4 \pi^{2} \Sigma x^{\Sigma} y^{\Sigma} x^{\prime} y^{\prime}$

In a dispersion-free straight section containing an undulacor, the effective size and the divergence of the beam, $\Sigma_{i}$ and $\Sigma_{i}{ }^{\prime}(i=x, y)$, are given by

$$
\Sigma_{i}=\left(\sigma_{\gamma}^{2}+\sigma_{i}^{2}\right)^{1 / 2} ; \Sigma_{i}^{\prime}=\left(\sigma_{\gamma}^{\prime 2}+\sigma_{i}^{2}\right)^{1 / 2}
$$

where $\sigma$ and $\sigma_{Y}^{\prime}$ are the source size and divergence of the radiation field, respectively. These are functions of the photon wavelength and undulator length $L$, and they include depth-of-field effects, diffraction effects, or both.

In Eq. 1, $\sigma_{i}$ and $\sigma_{i}^{\prime}$ are the positron beam size and divergence. These quantities are functions of the emittance of the lattice and the value of the $\beta$-functions in the straight sections. The values of the $\beta$-functions, which are normally optimized for large dynamic aperture of the storage ring, determine the stability of its operation. There are certain ranges of $B-$ function values that are suitable in the present design. The rms Gaussian width and divergence of the positron beam are given by 


$$
\sigma_{i}=\sqrt{\varepsilon_{i}{ }^{\beta}} ; \sigma_{i}^{\prime}=\sqrt{\varepsilon_{i} / \beta_{i}} \quad(i=x, y)
$$

where $\varepsilon_{x}=7.3 \mathrm{~nm}-\mathrm{rad}$ and $\varepsilon_{y}=0.73 \mathrm{~nm}-\mathrm{rad}$ are the positron beam emittance in the horizontal (orbital) plane and in the vertical plane, respectively.

In the hard $x$-ray wavelength range $(<10 \AA)$, the contribution to the size and divergence from the radiation field is negligible. Hence, for photons with greater than $1 \mathrm{keV}$ energy,

$$
\Sigma_{I}=\sigma_{i} ; \Sigma_{i}^{\prime}=\sigma_{i}^{\prime} \quad(i=x, y)
$$

The $\beta$-functions of the lattice then determine the size and divergence of the positron beam and, hence, that of the photon beam.

In Table 4.1, typical values of the size and divergence of bendingmagnet, undulator, and wiggler sources are given for a chosen set of Bfunctions.

From these values, an estimate of the number of photons striking a sample of given dimensions and at a distance $D$ from the source can be made. As an example, we choose a $0.5 \mathrm{~mm} \times 0.5 \mathrm{~mm}$ size sample with area $0.25 \mathrm{~mm}^{2}$ located at $40 \mathrm{~m}$ from the source. In this case, the sample subtends an angular width of $0.0125 \mathrm{mrad}$ in the horizontal and vertical directions, which is smaller than the effective angular width of the photon beam for any of the $\mathrm{X}$-ray sources discussed.

TABLE 4.1 Valueg of B-Functions in Different Parts of the Lattice and Dimensions of Different Sources $\left(\varepsilon_{x}=7.3 \mathrm{~nm}-\mathrm{rad} ; \varepsilon_{y}=0.73 \mathrm{~nm}\right.$-rad)

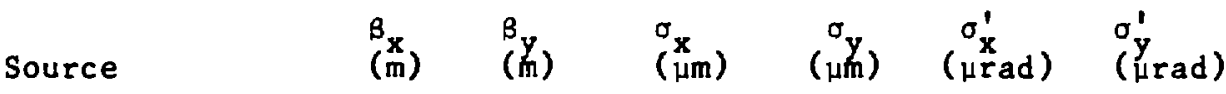

\begin{tabular}{lrrrrrr}
\hline Bending Magnet & 1.8 & 17.0 & 114 & 111 & 63 & 7 \\
Undulator 1 & 13.0 & 10.0 & 308 & 85 & 24 & 9 \\
Undulator 2 & 20.0 & 5.0 & 382 & 60 & 19 & 12 \\
Wiggler & 13.0 & 10.0 & 308 & 85 & 24 & 9 \\
\hline
\end{tabular}


For the undulator, the brilliance is assumed to be approximately Gaussian in shape, with the on-axis brilliance value as the amplitude and an effective source area given by $2 \pi \Sigma_{x} \Sigma_{y}$. The on-axis brightness is obtained by multiplying the on-axis brilliance by this area. The total number of photons $N$ on the sample is approximately $(0.0125 \mathrm{mrad})^{2}$ times the on-axis brightness, resulting in $\mathrm{N} \approx 1 \times 10^{14} \mathrm{ph} / \mathrm{s} \cdot 0.17 \mathrm{BW}$.

For the bending magnet and wiggler sources the number of photons striking the sample at the critical energy is given approximately by $(0.0125 \mathrm{mrad})^{2}$ times the on-axis brightness. In the case of wiggler $A$, using the brightness given in Fig. 7.2 (see Sec. 7), $N \approx 6.3 \times 10^{11} \mathrm{ph} / \mathrm{s} \cdot 0.1 \% \mathrm{BW}$, and for the bending magnet source, $\mathbb{N}=1.5 \times 10^{10} \mathrm{ph} / \mathrm{s} \cdot 0.1 \% \mathrm{BW}$.

The advantages of the highly collimated radiation from the undulator are clearly evident in this example. The total number of photons obtained with the undulator source is greater than 150 times that with the wiggler source.

In larger samples, with dimensions that subtend the entire vertical extent of the insertios-device beam and approximately 1 mrad of the horizontal extent of the wiggler beam, the number of photons on the sample from the undulator is only approximately three times that of the wiggler. The bending magnet in this case produces a factor of approximately 40 times less $\mathrm{flux}$ on the sample area. 


\section{BENDING-MAGNET SOURCES}

\subsection{APS BENDING-MAGLET CONFIGURATION}

The primary purpose of the 7-GeV APS is to provide radiation from the insertion devices. However, the facility also can deliver bending-magnet radiation that can be used to carry out preliminary studies of systems prior to more exhaustive studies using insertion-device radiation. The bending magnets can also be useful for experiments needing high brilliance or high photon energies.

The 7-GeV APS design includes 35 bending-magnet (BM) sources. Each bend covers $4.5^{\circ}(78.5 \mathrm{mrad})$, and the trajectory of the positrons through each bend has a radius of $38.96 \mathrm{~m}$. The vertical opening angle $2 \psi$ of this radiation sheet at the critical energy is approximately $1 / \gamma$, where $\gamma$ is the relativistic enhancement of the positron rest energy. For the $7-\mathrm{GeV}$ positrons, $\gamma$ is equal to 13,699 . Hence, the value of $1 / \gamma$ is about 73 urad. The constraints from the storage-ring vacuum chamber and crotch design allow an extraction of about $\pm 3 \mathrm{mrad}$ of radiation in the orbital plane.

Table 5.1 compares the parameters of BM sources on the 7-GeV APS with those of several operating hard $x$-ray storage rings. The 1 ist includes design parameters (which may not correspond to current operating parameters) for the National Synchrotron Light Source (NSLS) $x$-ray ring, located at Brookhaven National Laboratory (BNL) in New York; the Stanford Synctrotron Radiation Laboratory (SSRL), which uses the Stanford Positron-Electron Accelerator Ring (SPEAR) at Stanford University in California; and the Cornell High Energy Synchrotron Source (CHESS), which uses the Cornell Electron Storage Ring (CESR), located at Cornell University in New York.

\subsection{FLUX, BRIGHTNESS, AND BRILLIANCE}

In Figs. 5.1-5.3, we have used the parameters of Table 5.1 to compare the flux, central brightness, and brilliance, respectively, from the BMs on the APS and three other sources. It is to be expected that the APS should have the highest flux for energies above about $10 \mathrm{keV}$; moreover, the APS BMs have the highest central brilliance over the whole spectral region. 


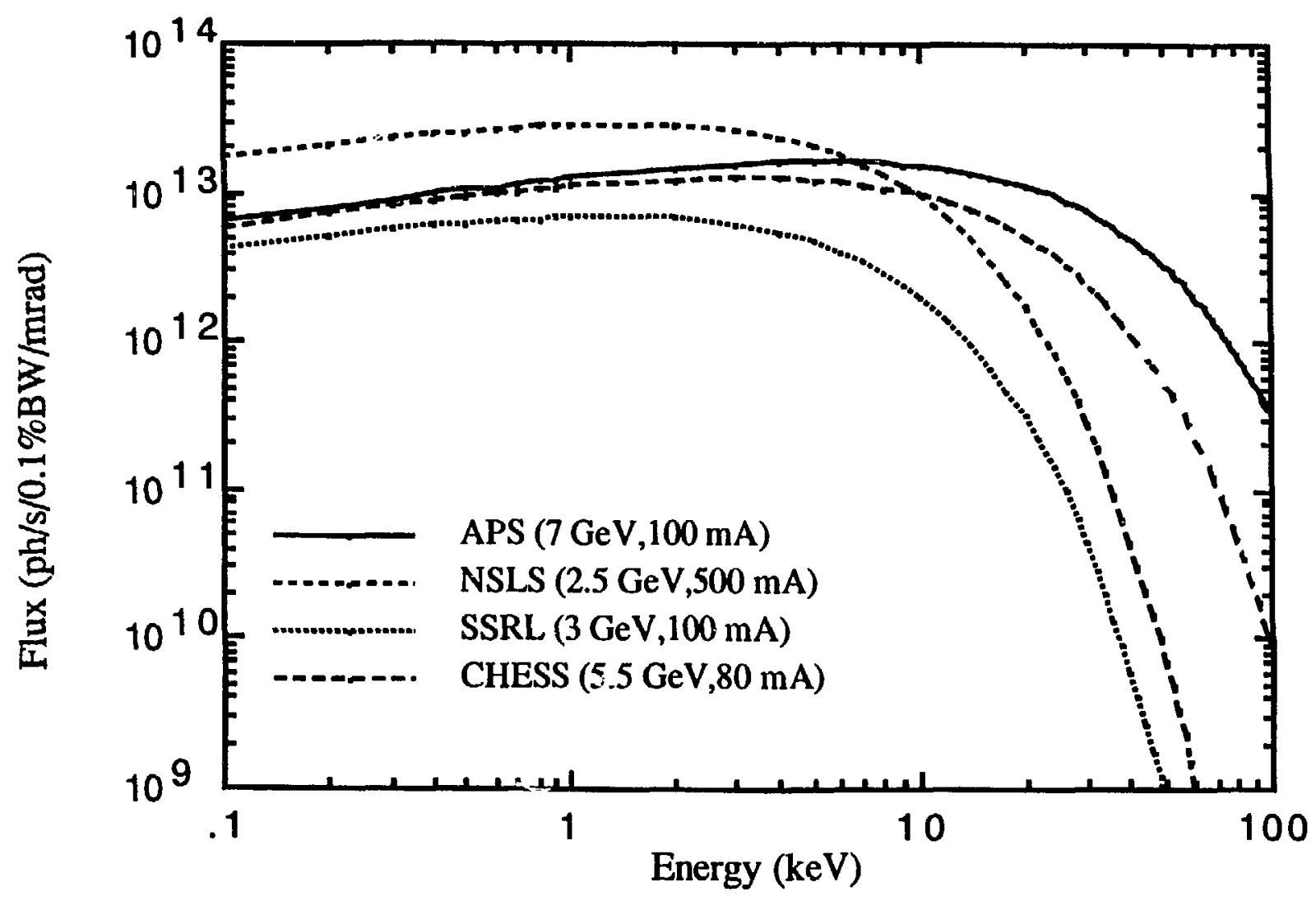

FIGURE 5.1 Flux from a Bending Magnet on Various Synchrotron Sources 


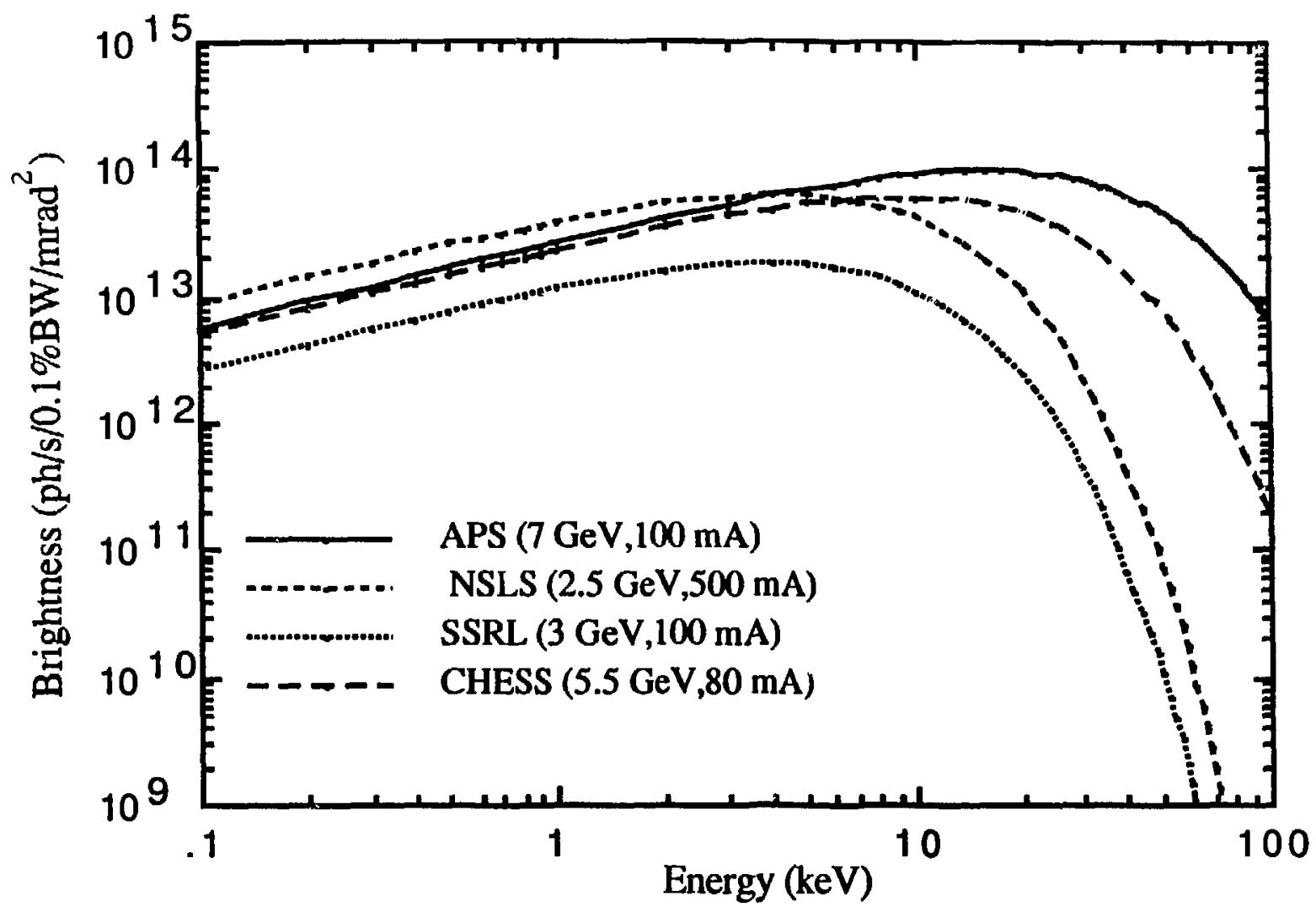

FIGURE 5.2 Brightness of Bending-Magnet Radiation from Various Synchrotron Sources 


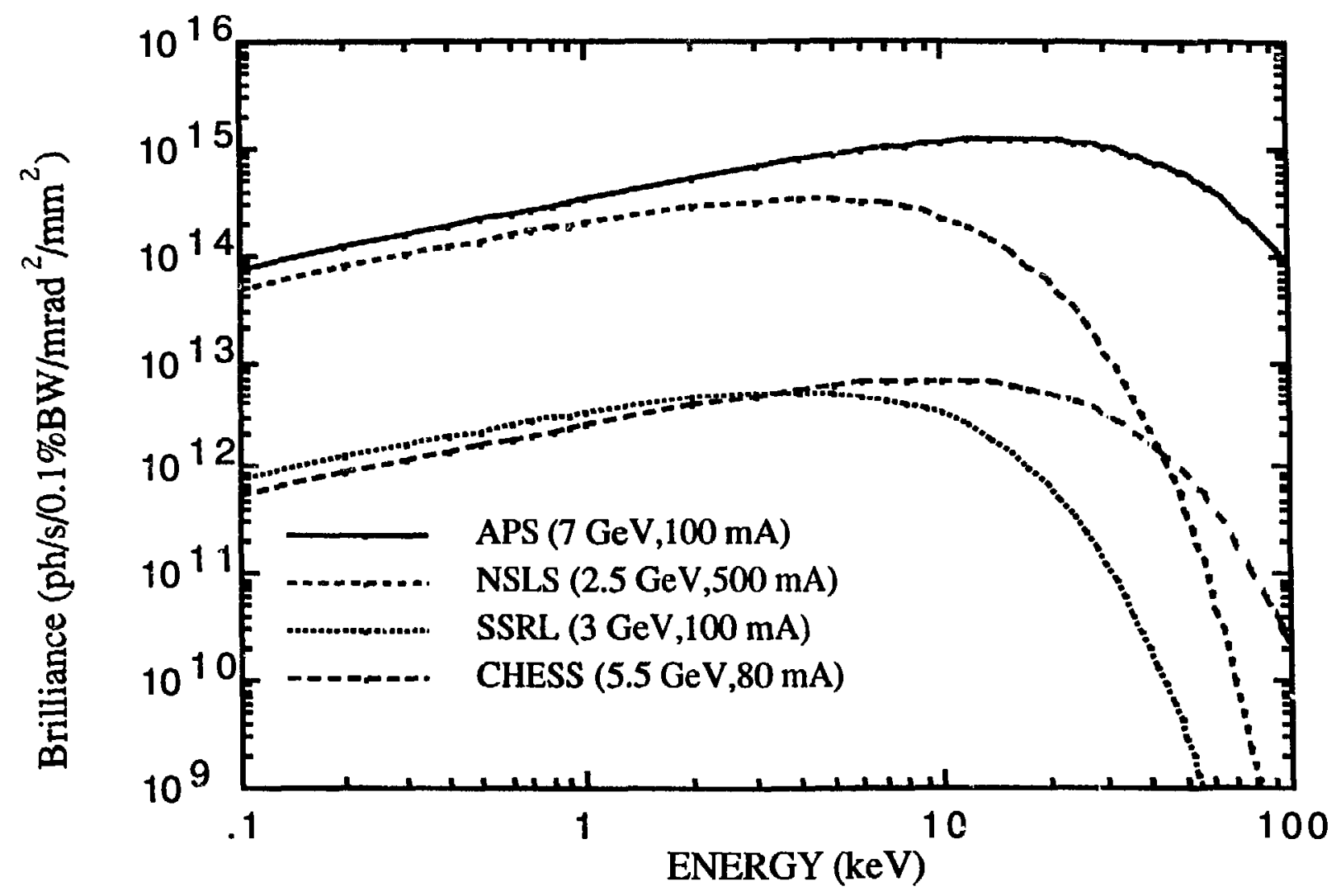

FIGURE 5.3 Brilliance of Bending-Magnet Kadiation from Various Synchrotron Sources 
TABLE 5.1 Parameters of Various Bending-Magnet Sources

\begin{tabular}{|c|c|c|c|c|}
\hline \multirow[b]{2}{*}{ Parameter } & \multicolumn{4}{|c|}{ Source } \\
\hline & APS & NSLS & SSRL & CHESS \\
\hline 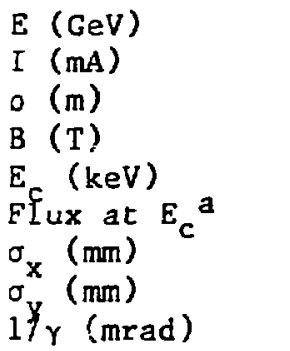 & $\begin{array}{l}7.0 \\
100 \\
38.96 \\
0.599 \\
19.5 \\
1.12 \\
0.11 \\
0.11 \\
0.073\end{array}$ & $\begin{array}{l}2.5 \\
500 \\
6.8 \\
1.22 \\
5.1 \\
0.4 \\
0.3 \\
0.1 \\
0.204\end{array}$ & $\begin{array}{l}3.0 \\
100 \\
12.7 \\
0.79 \\
4.7 \\
0.48 \\
2.0 \\
0.3 \\
0.170\end{array}$ & $\begin{array}{l}5.5 \\
80 \\
32.0 \\
0.57 \\
11.5 \\
0.88 \\
1.4 \\
1.0 \\
0.093\end{array}$ \\
\hline
\end{tabular}

${ }^{\mathrm{a}}$ In units of $10^{13}$ photons $/ \mathrm{s} \cdot 0.1 \% \mathrm{BW} \cdot \mathrm{mrad}$ 


\section{UNDULATOR SOURCES}

\subsection{UNDULATOR RADIATION ENERGY}

An undulator has many properties that make it an extremely attractive source of electromagnetic radiation. In particular, the radiation is concentrated in a number of narrow pseudo-monochromatic energy bands known as the harmonics of the device. Another useful property is that, under favorable operating conditions, the energy of these harmonics can be shifted or "tuned" over an energy interval that can be as large as two or three times the value of the first harmonic.

The most prominent feature of the 7-GeV Advanced Photon Source design is the large number of straight sections for such undulators. The emittance of the present lattice has been minimized and the $B$-functions have been made large in the straight sections to provide a low-divergence positron beam. To obtain narrow pseudo-monochromatic harmonics from an undulator, it is essential that the beam divergence be comparable with or smaller than the maximum angle of undulations in the device.

The photon energy (in $\mathrm{keV}$ ) of the nth harmonic at an observation angle $\theta$ (in racians) relative to the undulator axis is given by

$$
E_{n}=\frac{0.949 E_{R}^{2} n}{\lambda_{0}\left(1+K^{2} / 2+\gamma^{2} \theta^{2}\right)}
$$

where $E_{R}$ is the positron energy in $\mathrm{GeV}$ and $\lambda_{0}$ is the undulator period in $\mathrm{cm}$. The deflection parameter $K$ is $g$ iven in terms of $\lambda_{0}$ and the peak magnetic field $B_{0}(\mathrm{~T})$ by

$$
K=0.934 \lambda_{0} B_{0}
$$

For the Nd-Fe-B hybrid magnet configuration with vanadium permendur pole pieces, $B_{0}$ is given by

$$
B_{0}=0.95 \times 3.44 \exp [-R(5.08-1.54 R)]
$$

where $R=G / \lambda_{0}$ and $G$ is the undulator gap in $\mathrm{cm}$. In $E q .6$, the factor 0.95 represents the "filling factor," which takes into account the packing factor of high-permeability blocks in the undulator assembly. Equation 6 applies to an optimized magnet configuration at a given $R$ and is valid for $0.07 \leq R \leq$ 0.7 , and only approximately valid for $0.7<R \leq 1$. Note that the smallest gap produces the largest value of $K$ and the lowest value of $E_{n}$.

In Table 6.1, various capabilities are indicated for three undulators with different periods planned for the 7-GeV APS. Their important features are as follows: 
- The undulators on the APS, built with available $\mathrm{Nd}-\mathrm{Fe}-\mathrm{B}$ hybrid magnet cechnology, deliver first-harmonic radiation with energies ranging from a tew hundred $\mathrm{eV}$ to about 25 $\mathrm{keV}$. The typical on-axis brilliance from the full emittance of the source for the first harmonic ranges from $10^{18}$ to $10^{19}$ photons/s.0.1\% $\mathrm{BW} \cdot \mathrm{mrad}^{2} \cdot \mathrm{mm}^{2}$.

- A fully tunable undulator with $3.3-\mathrm{cm}$ period on the APS will deliver radiation from 4 to $13 \mathrm{keV}$ in the first harmonic, and from 12 to $40 \mathrm{keV}$ in the third harmonic. The energy tunability is achieved by varying the gap between 1.0 and $2.8 \mathrm{~cm}$.

- For applications needing only the high-energy undulator First-harmonic radiation, a device with $2.3-\mathrm{cm}$ period will be tunable from 13 to $20 \mathrm{keV}$ through gap variation. The undulator with a period of $20 \mathrm{~cm}$ provides tunability in the soft $x$-ray range.

- The vacuum chambers for the undulators will permit a minimum gap of either 1.4 or $1.0 \mathrm{~cm}$. The former chamber will be used during the initial operation of the APS.

- Within the currently projected scope of research at the APS, there will be little demand for harmonics higher than the third. The required magnet design tolerances for such undulator sources are well within those achievable with present-day technology. A prototype of the $3.3-\mathrm{cm}$ device is now being built; its performance will be tested at CHESS.

TABLE 6.1 Characteristics of Three Selected Undulators on the 7-GeV APS

\begin{tabular}{lccc}
\hline Parameter & Undulator $A$ & Undulator B & Undulator C \\
\hline Period $(\mathrm{cm})$ & 3.3 & 2.3 & 20 \\
$\mathrm{~K}_{\max } / \mathrm{K}_{\min }$ & $2.5 / 0.4$ & $1.1 / 0.3$ & $2.7 / 1.0$ \\
$\mathrm{G}_{\min } / \mathrm{G}_{\max }(\mathrm{cm})$ & $1.0 / 2.8$ & $1.0 / 2.0$ & $16 / 27$ \\
$\mathrm{E}_{\min } / \mathrm{E}_{\max }(\mathrm{keV})$ & $3.5 / 13.5$ & $13.0 / 19.5$ & $0.5 / 1.5$ \\
\hline
\end{tabular}




\subsection{SPECTRAL CHARACTERISTICS}

For a single particle moving through the periodic field of a transverse undulator, the energy spectrum and the angular dependence of the spectral brightness of the undulator can be calculated using analytical expressions valid for small values of $\mathrm{K}$. Since we are dealing with a positron beam with non-zero emittance, it is best to obtain spectral characteristics using numerical methods. Our method is based on an integration of the LienardWiechert potential over a positron trajectory of finite dimension. 15 These calculations are computer-intensive, particularly for the calculations of the flux through a pin-hole, which demands additional integration.

Undulator A, with a period of $3.3 \mathrm{~cm}$, satisfies the user criteria of providing first-harmonic tunability over the energy range from 7 to $14 \mathrm{keV}$ during early operation, and from 3.5 to $13.5 \mathrm{keV}$ during mature operation. A two-meter-long prototype of this device has now been built and will be tested on the CESR lattice at Cornell. 16 The on-axis brilliance of this device has been shown in Fig. 6.1 for $G=1.3 \mathrm{~cm}, K=1.65$. In this configuration, the first harmonic will occur at about $6 \mathrm{keV}$ and the expected third harmonic at about $18 \mathrm{keV}$. The second harmonic (at about $12 \mathrm{keV}$ ) also has considerable onaxis brilliance. This arises because we are dealing with a positron source with non-zero emittance, so that the angular dependence of the photon energy expressed in Eq. 4 can produce even harmonics.

In Fig. 6.2, a set of first-harmonic peaks produced by varying the gap of Undulator $A$ is shown. The figure demonstrates the tunability of this device, as well as the rather weak dependence of brilliance on photon energy. Similar results for a 2.3-cm-period undulator (B) are shown in Fig. 6.3. This device produces first-harmonic radiation with higher photon energy but somewhat smaller tunability range. In Fig 6.4, two spectra for the device with 20-cmperiod are shown. This device, with a first-harmonic tunability from 0.5 to $1.5 \mathrm{keV}$, is most suitable for investigations needing soft $X$-ray radiation. (The 7.7-cm-period pure REC undulators, installed on the 14-GeV PEP lattice to obtain hard $X$-rays, will produce tunable first-harmonic radiation covering the 1 to $4 \mathrm{keV}$ range on the 7-GeV APS lattice.)

The angular distribution of radiation from an undulator is dependent on the harmonic. Several distributions for Undulator A are shown; note that the source emittance is included in our calculations. In Fig. 6.5, the variations of the $\sigma$ - and $\pi$-components of polarization contributing to the undulator brilliance as a function of horizontal angle, $\theta$ (for $\psi=0$ ), and vertical angle, $\psi$ (for $\theta=0$ ), are showis. These represent the variation along the $x$ axis and y-axis, respectively. From this figure, we observe that along the $x-$ axis there is only a contribution from the o-component of the polarization. Along the $y$-axis, the $\pi$-component is very small along the undulator axis but increases away from the axis. Also, we observe contributions to the bril1 iance as peaks at various values of $\theta$ arising from higher harmonics, as predicted by Eq. 4. The figure shows that the nature of the polarization of radiation emitted by a planar undulator is dependent on $\theta$ and $\psi$. The inclusion of the positron beam emittance smears the polarization components with respect to those predicted by a single-positron calculation. However, as Fig. 6.5 shows, the $\pi$ and $\sigma$ components of polarization are clearly observable. The calculations suggest the possibility of selecting radiation with different ellipticity by choosing an appropriate angle of nbservation $(\theta, \psi)$ for any undulator energy harmonic. 


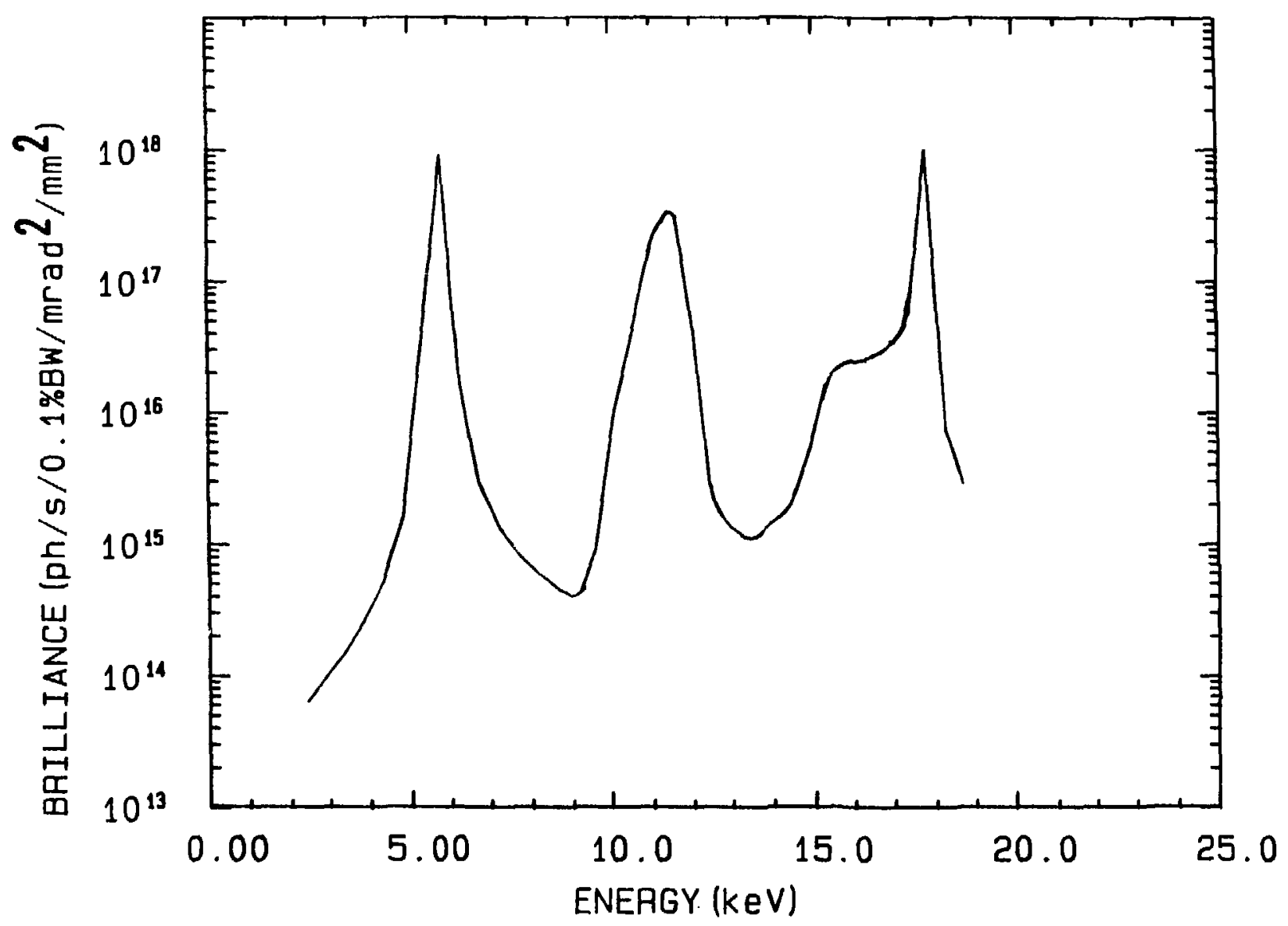

FIGURE 6.1 On-Axis Brilliance vs. Energy for APS Undulator A at $100 \mathrm{~mA}$, Showing the First Three Radiation Harwonics (The Monte Carlo calculations include the positron phase-space dimensions.) 


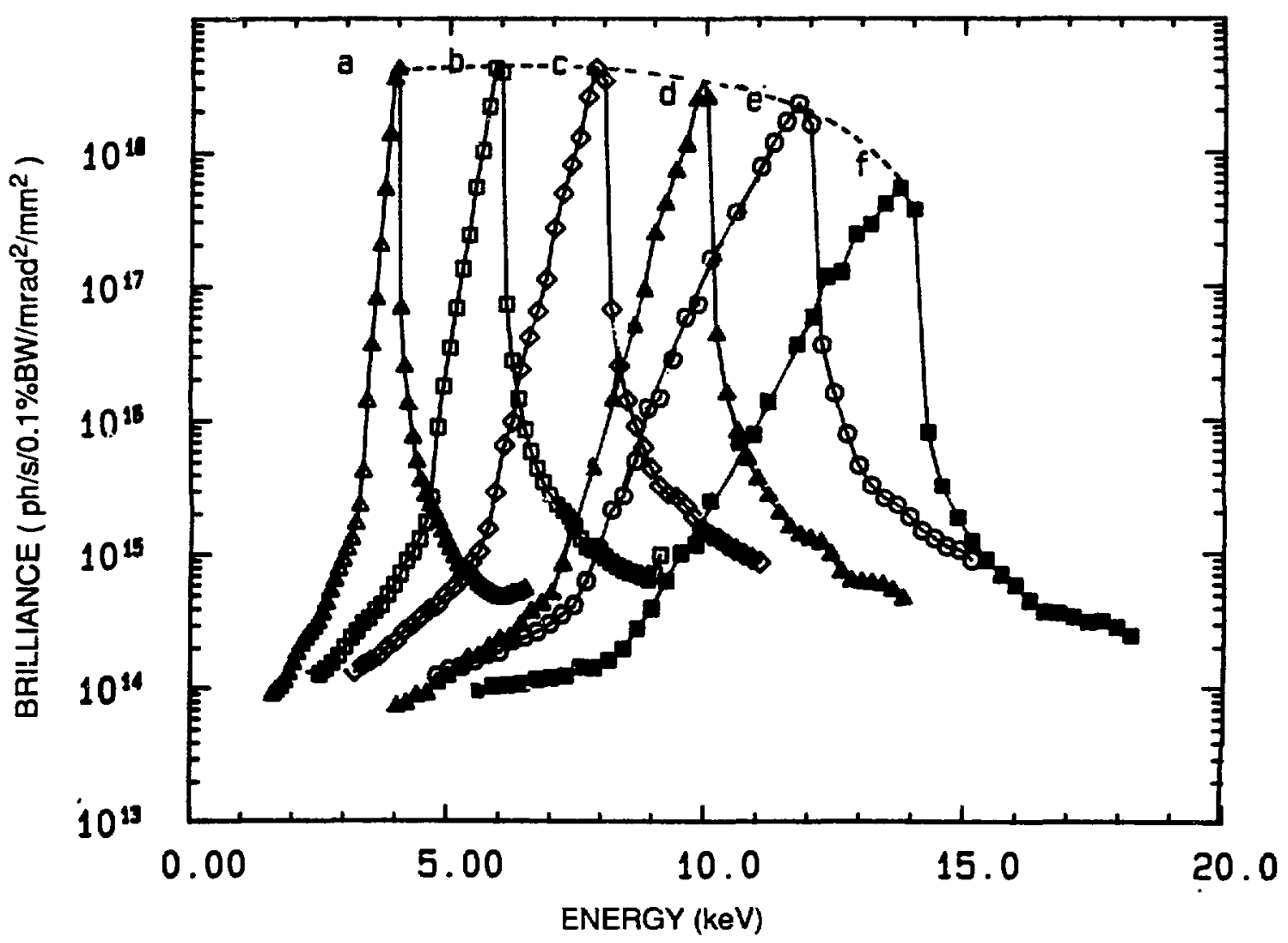

PIGURE 6.2 On-Aris Brilliance of the First-Harmonic Radiation from APS Undulator $A$ at $7 \mathrm{GeV}$ and $100 \mathrm{~mA}$ (The first-harmonic peaks at various energies are obtained at agnet gap settings of (a) $11.2 \mathrm{~mm}$, (b) $13.9 \mathrm{~mm}$, (c) $16.5 \mathrm{~mm}$, (d) $19.7 \mathrm{~m}$, (e) $24.7 \mathrm{~mm}$, and (f) $30.1 \mathrm{~mm}$. These calculations include the phase-space dimensions of the positron beam.) 


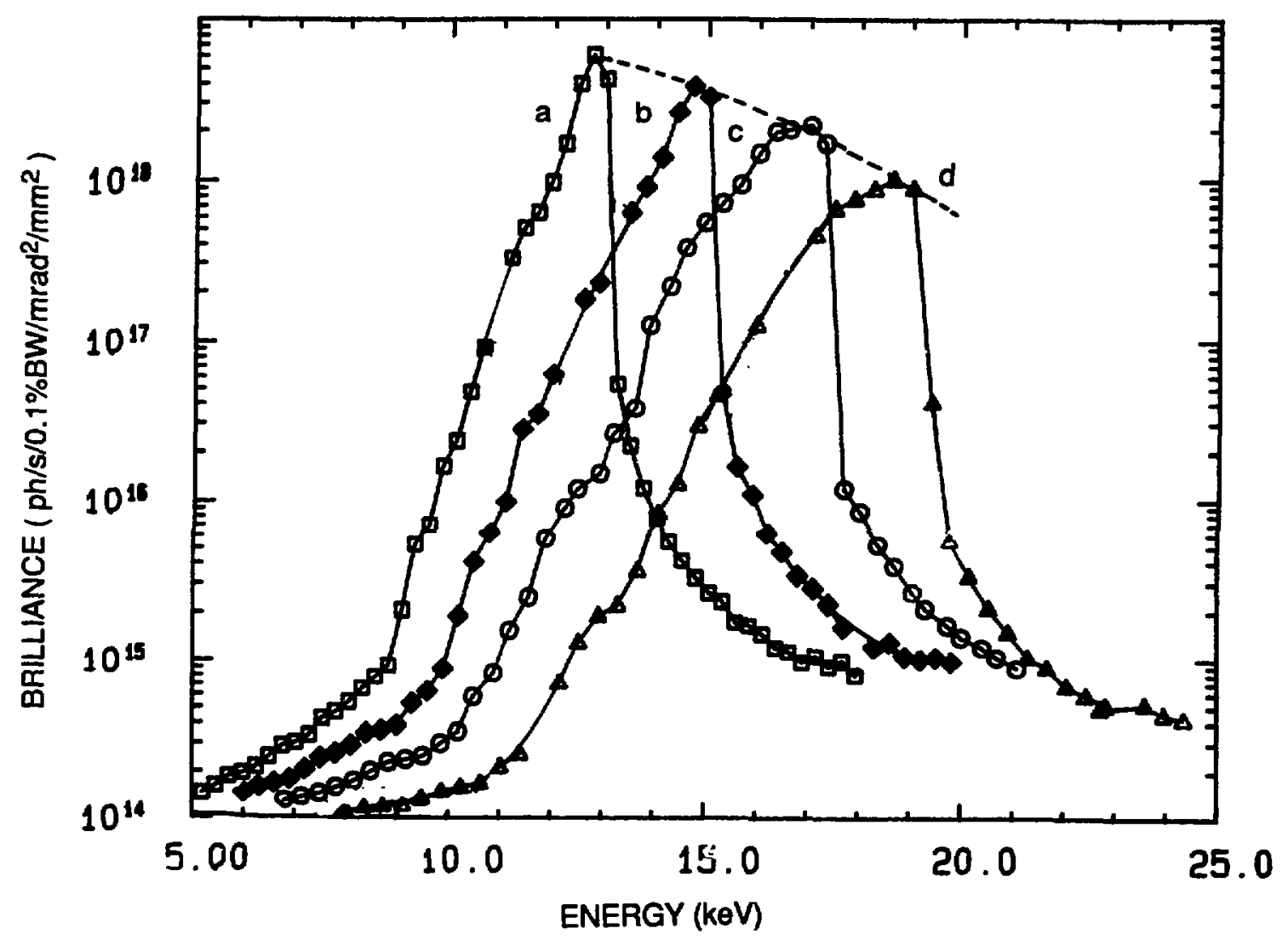

FIGURE 6.3 On-Axis Brilliance of the First-Harwonic Radiation from APS Undulator $B$ at $7 \mathrm{GeV}$ and $100 \mathrm{aA}$ (The first-harmonic peaks at various energies are obtained at magnet gap settings of (a) $10 \mathrm{~m}$, (b) $11.5 \mathrm{~m}$, (c) $13.5 \mathrm{~m}$, and (d) $17.5 \mathrm{~mm}$. These calculations include the phase-spece dimensions of the positron beam.) 


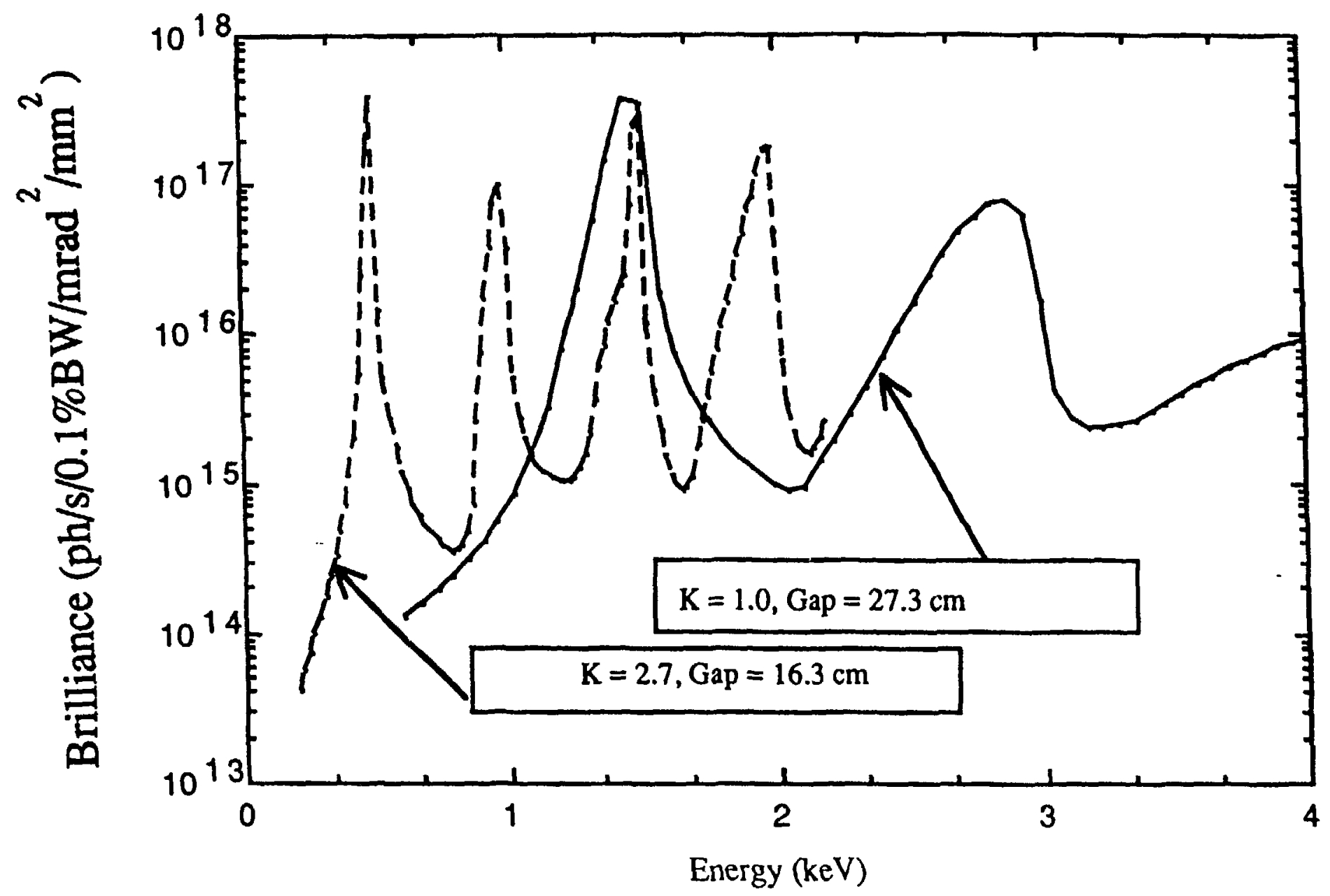

FIGURE 6.4 On-Axis Brilliance from APS Undulator C at $7 \mathrm{GeV}$ and $100 \mathrm{~mA}$ (The two energy spectra covering the soft x-ray range are obtained by altering the gap from $16 \mathrm{~cm} \mathrm{to} 27 \mathrm{~cm}$. These calculations include the phase-space dimensions of the positron beam.) 

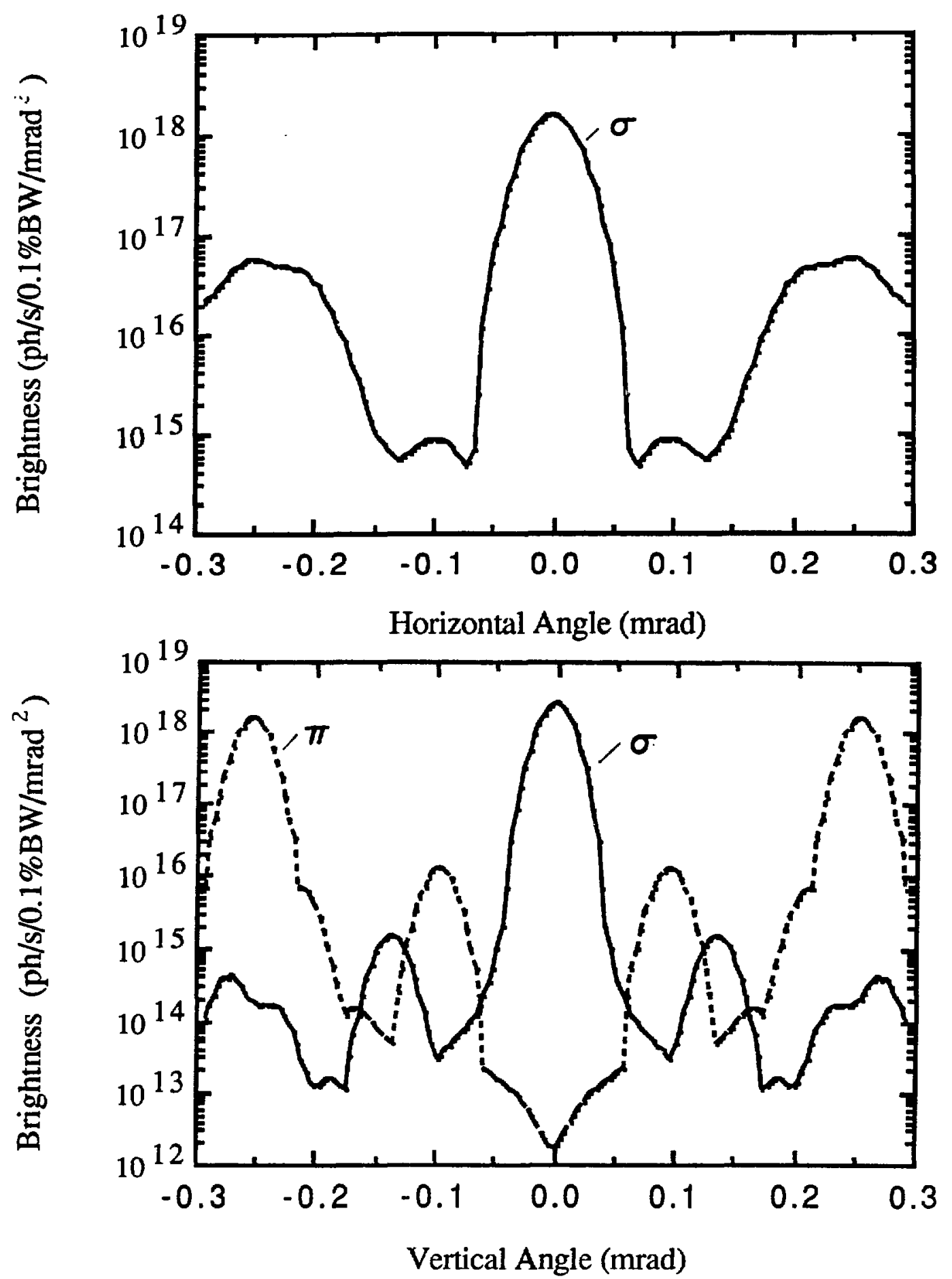

FIGURE 6.5 Angular Distribution of the First-Harmonic Radiation from APS Undulator $A$ along $x-$ and $y$-Axes, Showing the $\sigma-$ and $\pi$ Components (Along the $x$-axis, there is only a o-component. The calculations include the phase-space dimensions of the positron beam.) 


\subsection{SEGYENTED UNDIJATOR}

Eor an idealized single-particle (zero-emittance) beam, one expects the brightness to be proportional to $\mathrm{N}^{\mathbf{X}}$, where $\mathrm{N}$ is the number of periods and $x=2$. This expectation has encouraged the design of long undulators with many periods to enhance the brilliance. At the same time, the detrimental effects on the brightness from the magnetic-field-errors require tight construction tolerances on the undulator assembly. For a non-zero-emittance lattice, a numerical calculation of the type discussed above has shown that the brighţness varies with $1<x<2$. For the APS undulators, we find that $\mathrm{x}=1.2 .17$

This result leads us to simplify the engineering design of the APS undulators by segmenting them and mounting the segments together. Thus, instead of a single 5-m-long undulator, one can design two undulators with approximately half the length. For Undulator A, segmentation into two short devices reduces the brightness by about 13\%. However, the segmenting provides the following advantages:

- The mechanical design of the undulator vacuum chamber is simplified, making it easier to achieve the required tolerance.

- Magnetic measurements on the segmented undulator are considerably easier.

- Individual segments can be separately tested for their performance.

- The effects of random errors on the peak brightness are smaller for the shorter (or segmented) devices.

- The power load on the first optics can be adjusted for its best performance by selecting the number of segments. This capability provides needed flexibility and experience in the optics design.

- The prototype undulator being built for CHESS, which is $2 \mathrm{~m}$ long, will provide all the required test information.

\subsection{BLUX THROUGH A PIN-HOLE}

The flux through a pin-hole placed on the axis of the undulator is calculated using the numerical procedures described earlier. These are complex and demand considerable computational time. In Fig. 6.6, a set of first-harmonic energy spectra from Undulator A (period $=3.3 \mathrm{~cm}, \mathrm{gap}=1.6 \mathrm{~cm}$ ) obtainable through pin-holes of different sizes is shown. The spectral distribution is a function of the pin-hole size. However, a pin-hole alone cannot be used as a monochromator, except in those investigations where one is interested in only a pseudo-monochromatic beam. The variation of flux through a pin-hole of fixed size placed on the axis of Undulator A as a function of 


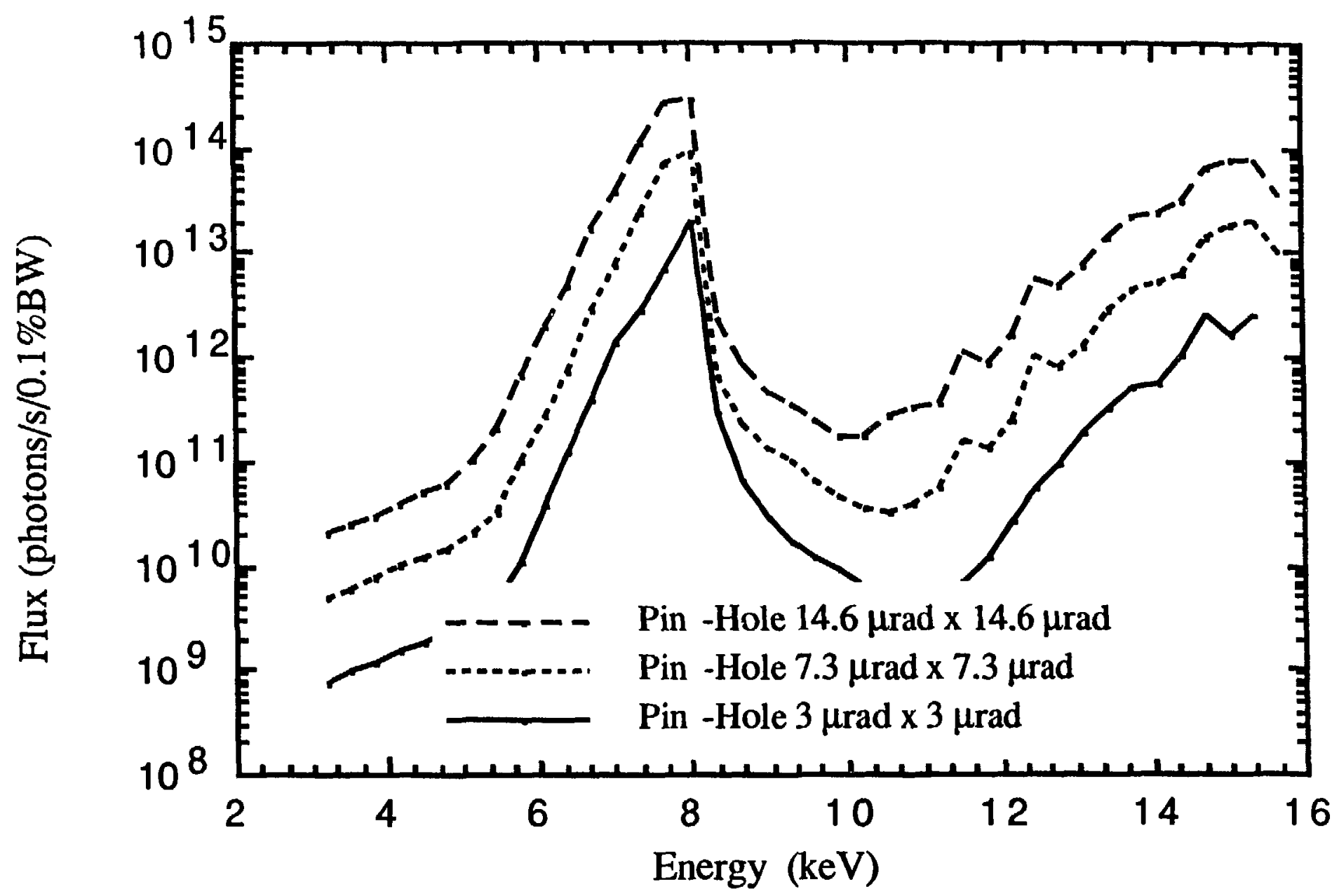

FICURE 6.6 Flux through a Set of Pin-Holes Showing the First-Harmonic Energy Spectra from APS Undulator A at $7 \mathrm{GeV}$ and $100 \mathrm{~mA}$ (The gap setting is $16 \mathrm{~mm}$. ) 
the first-harmonic $x$-ray energy is shown in Fig. 6.7 . At $30 \mathrm{~m}$ from the center of the undulator, this pin hole illuminates an area of $0.66 \mathrm{~mm} \times 0.66 \mathrm{~mm}$. The effective opening angle of the central cone is approximately 9 urad in the vertical direction and $24 \mu \mathrm{rad}$ in the horizontal direction.

\subsection{ENERGY SPREAD IN UNDULATOR PEARS}

For an undulator with $\mathrm{N}$ periods, the natural emitted bandwidth of the emitted radiation for an ideally parallel positron beam is given by

$$
\Delta_{1}=\frac{\Delta E_{n}}{E_{n}}=\frac{1}{n N},
$$

where $\mathfrak{n}$ is the radiation harmonic. In the case of the proposed Undulator $A$, $\mathrm{N}=158$ and the bandwidth of the first harmonic is approximately $0.6 \%$.

In the case of the APS storage ring with non-zero emittance, there are other sources of energy spread. The contribution to the intrinsic bandwidth from the angular spread of the positron beam is

$$
\Delta_{2}^{i}=\left(\sigma_{i}^{i} \gamma\right)^{2} /\left(1+k^{2} / 2\right), \quad i=x, y .
$$

For an undulator straight section in the present lattice, we have (from Table 4.1) $\sigma^{\prime}=24 \mu \mathrm{rad}$ and $\sigma^{\prime}=9 \mu \mathrm{rad}$. For a typical value of $k=1.0$, the energy ${ }^{x}$ spreads along the $x$ and $y$ directions are 6.6 and $0.9 \%$, respectively. The broadening effects along the two directions are not equal, and correspond to different effective opening angles of the central radiation cone in the horizontal and vertical directions.

The size of the positron beam also contributes to the energy spread. This can be estimated for a typical beam-line length of $M$ meters, using the expression

$$
\Delta_{3}^{i}=\left(\sigma_{i} / M\right)^{2} \gamma^{2}, \quad i=x, y .
$$

For our lattice, with $\sigma_{x}=308 \mu \mathrm{m}$ and $\sigma_{y}=85 \mu \mathrm{h}$, the bandwidths along the $x$ and $y$ directions will be 0.5 and $0.04 \%$, respectively. This spread is small compared with that produced by the beam divergence.

The spread in the energy of the positron beam will also contribute to the bandwidth. In the case of the APS lattice, this is less than $0.1 \%$.

A final source of undulator peak broadening is associated with inhomogeneities in the magnetic field of the undulator. For a variation in the peak field ( $B_{0}$ ) given by $\Delta B_{0}$ along the length of the device, the increase in the bandwidth is given by 


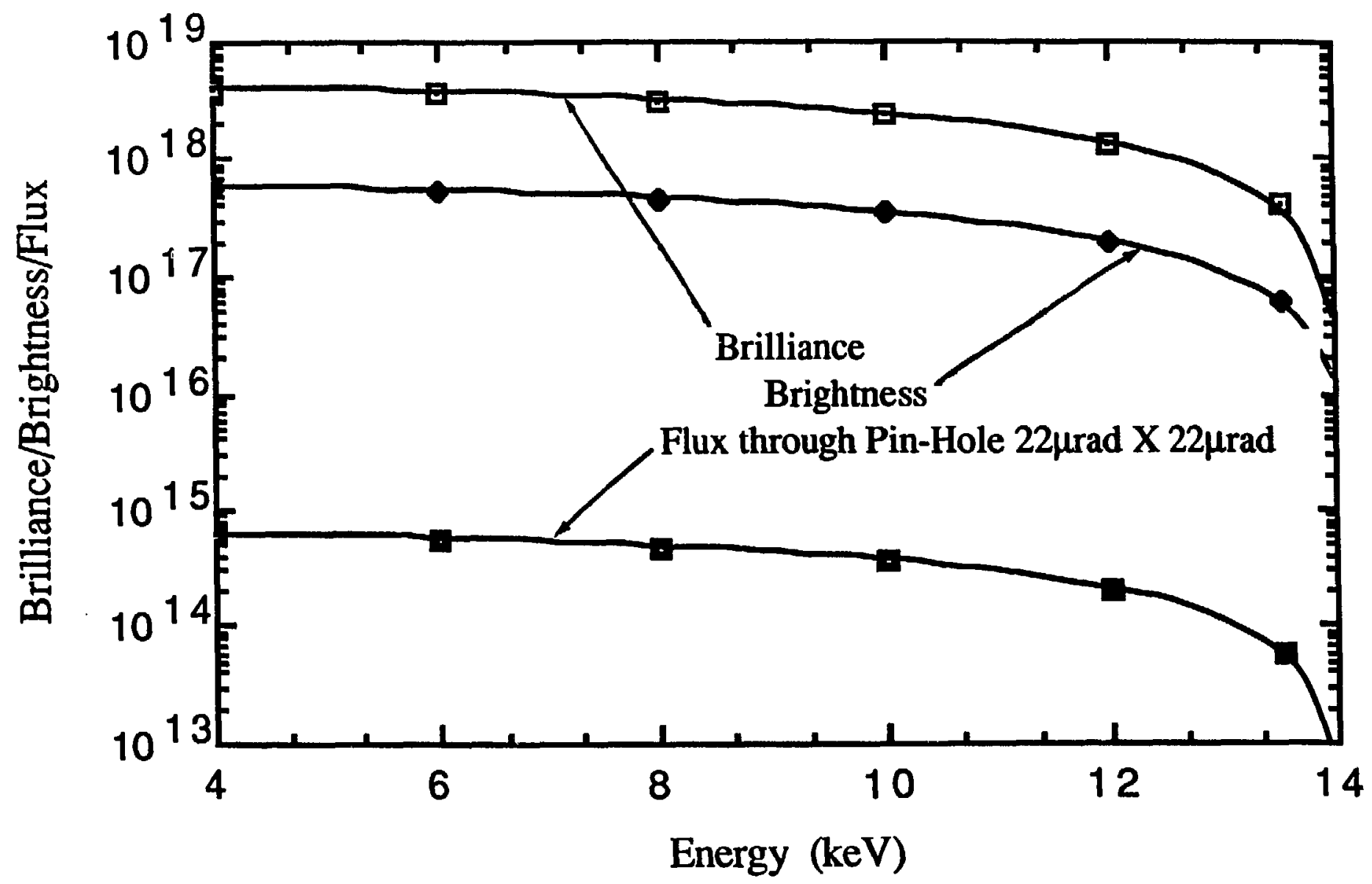

FIGURE 6.7 On-Axis Brillance, Brightness, and Flux through a Pin-Hole of Size $22 \mu \mathrm{rad} \times 22 \mu \mathrm{rad}$ Placed along the Axis of Undulator A vs. X-Ray Energy of the First Harmonic 


$$
\Delta_{4}^{i}=\left(\Delta B_{0} / B_{0}\right) \frac{K^{2}}{\left(1+K^{2 / 2}\right)}
$$

For the undulators proposed for the APS, the field errors are less than $0.5 \%$ and will result in a similar increase in the energy bandpass of the device.

The total energy bandpass is given approximately by the sum of the various contributions mentioned above. The energy spread from the undulators on the APS is on the order of $2 \%$ over the effective vertical opening angle and approximately 97 over the horizontal angle. 


\section{WIGGLER SOURCES}

In a wiggler, the positrons experience large magnetic fields such that $K>5$, and their large path-length deviations from a straight 1 ine do not permit the radiation to add coherently. Also, the large $K$ value in Eq. 4 produces very close harmonics, resulting in a nearly continuous spectral distribution resembling that from a bending magnet. The wigglers serve two important purposes on the 7-GeV APS. First, they can be used to set the critical energy of the spectral distribution higher or lower than that from the bending magnet. Second, by having a large number of poles in a 5-m-long multipole wiggler the spectral intensity can be propoztionally increased. The opening angles of radiation for a wiggler are considerably larger than those for an undulator. Thus, the spectral brilliance (or even the flux through a small pin hole) from a wiggler is lower than that from the first harmonic of an undulator. The primary uses of a wiggler on the APS will be to generate photons with energy above about : $0 \mathrm{keV}$ and in cases where one can use the entire angular spread of a wiggler beam. There will also be a need for wigglers with low critical energy, where higher harmonics of radiation are hard to eliminate.

In Table 7.1, parameters for two wigglers on the 7-GeV APS are given. Wiggler A has a critical energy of $35 \mathrm{keV}$, while Wiggler B has a low critical energy, approximately half that of the bending magnet. Wiggler A can be assembled with permanent magnets in a hybrid configuration, while Wiggler $B$ is best constructed from electromagnets. Note that Wiggler A can provide high brilliance even at $80 \mathrm{keV}$.

For producing photons in the range of 50 to $200 \mathrm{keV}--$ useful for performing Compton scattering and high $Q$ scattering experiments - a wiggler with $\mathrm{Nd}-\mathrm{Fe}-\mathrm{B}$ hybrid configuration has been designed. This wiggler has a period of $12 \mathrm{~cm}$ and a critical energy of $58 \mathrm{keV}$.

It is useful to compare the performance of the 7-GeV APS wigglers with those operating on or planned for other storage rings. In Figs. 7.1, 7.2, and 7.3 , we present the dependence of flux, brightness and brilliance, respectively, for various wigglers as a functiun of photon energy. In generating these figures, the parameters given in Table 7.1 have been used. 
Table 7.1 Parameters for Various Wiggler Sources

\begin{tabular}{|c|c|c|c|c|c|}
\hline Parameter & $\begin{array}{c}\text { APS } \\
\text { Wiggler A }\end{array}$ & $\begin{array}{c}\text { APS } \\
\text { wiggler B }\end{array}$ & $\begin{array}{r}\text { CHESS } \\
\text { West }\end{array}$ & $\begin{array}{l}\text { SSRL } \\
\text { VI }\end{array}$ & $\begin{array}{l}\text { NSLS } \\
x-17\end{array}$ \\
\hline$E_{R}(G e V)$ & 7.0 & 7.0 & 5.5 & 3.0 & 2.5 \\
\hline$I(m s)$ & 100 & 100 & 80 & 100 & 500 \\
\hline$\rho(m)$ & 23.3 & 78.0 & 13.2 & 8.3 & 1.4 \\
\hline$B_{0}(T)$ & 1.0 & 0.3 & 1.39 & 1.2 & 6.0 \\
\hline$x(\mathrm{~mm})$ & 0.31 & 0.31 & 1.9 & 2.5 & 0.3 \\
\hline$y(m m)$ & 0.09 & 0.09 & 1.21 & 0.15 & 0.02 \\
\hline$E_{c}(k e V)$ & 32.6 & 9.8 & 27.9 & 7.16 & 24.9 \\
\hline Poles & 20 & 40 & 6 & 54 & 6 \\
\hline Period $(\mathrm{cm})$ & 15 & 25 & 35 & 7.25 & 17.8 \\
\hline $\mathbf{K}$ & 14 & 7 & 45 & 8 & 99 \\
\hline$L(m)$ & 1.5 & 5.0 & 1.05 & 1.9 & 0.53 \\
\hline Structure & $\begin{array}{l}\text { Nd-Fe-B } \\
\text { hybrid }\end{array}$ & $\begin{array}{l}\text { Electro- } \\
\text { magnet }\end{array}$ & $\begin{array}{l}\text { Electro- } \\
\text { magnet }\end{array}$ & $\begin{array}{l}\text { Sm-Co } \\
\text { hybrid }\end{array}$ & $\begin{array}{l}\text { Super- } \\
\text { conducting }\end{array}$ \\
\hline $2 \theta(\operatorname{mrad})$ & 2.0 & 1.0 & 8.4 & 2.8 & 40.8 \\
\hline Flux at $E_{c}^{a}$ & 2.24 & 4.48 & 0.53 & 2.59 & 0.24 \\
\hline
\end{tabular}

${ }^{a}$ In units of $10^{14}$ photons $/ \mathrm{s} \cdot 0.1 \% \mathrm{BW} \cdot \mathrm{mrad}$. 


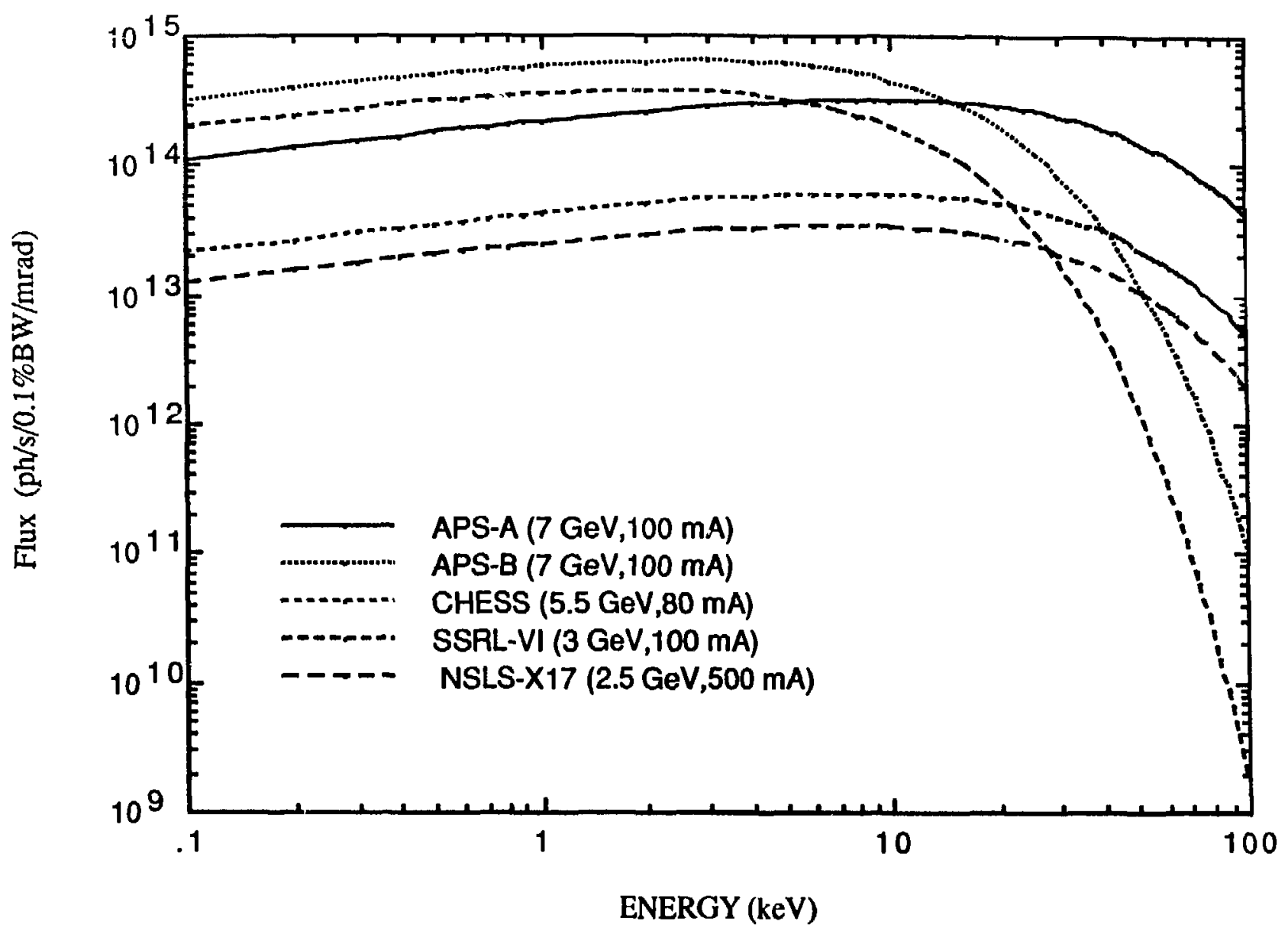

FIGURE 7.1 Flux from Wigglers on the 7-GeV APS at $100 \mathrm{~mA}$, Compared with Flux from Wigglers on Other Synchrotron Sources 


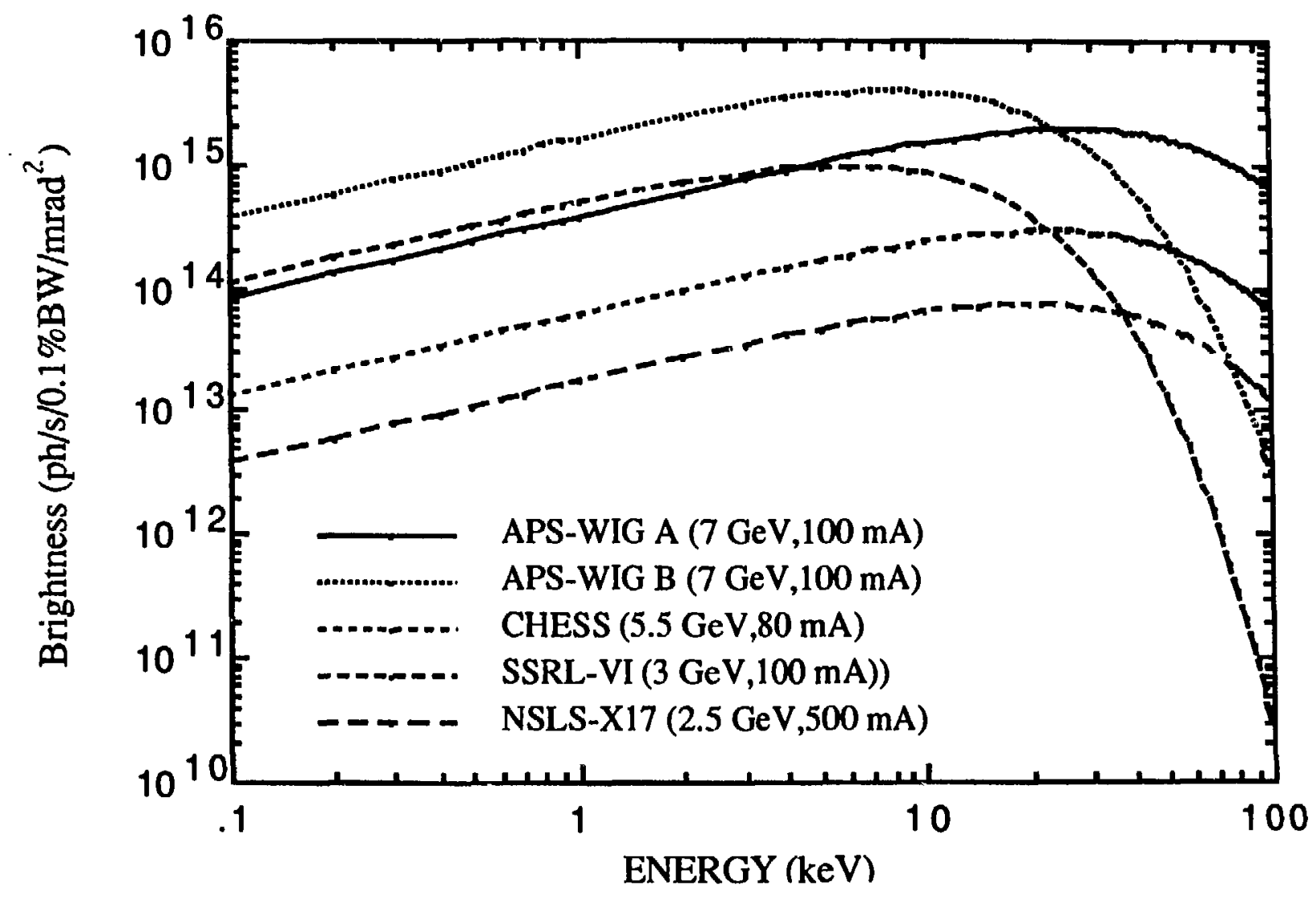

FIGURE 7.2 Central Brightness from Wigglers on the 7-GeV APS at $100 \mathrm{~mA}$, Compared with That frow Wigglers on Other Synchrotron Sources 


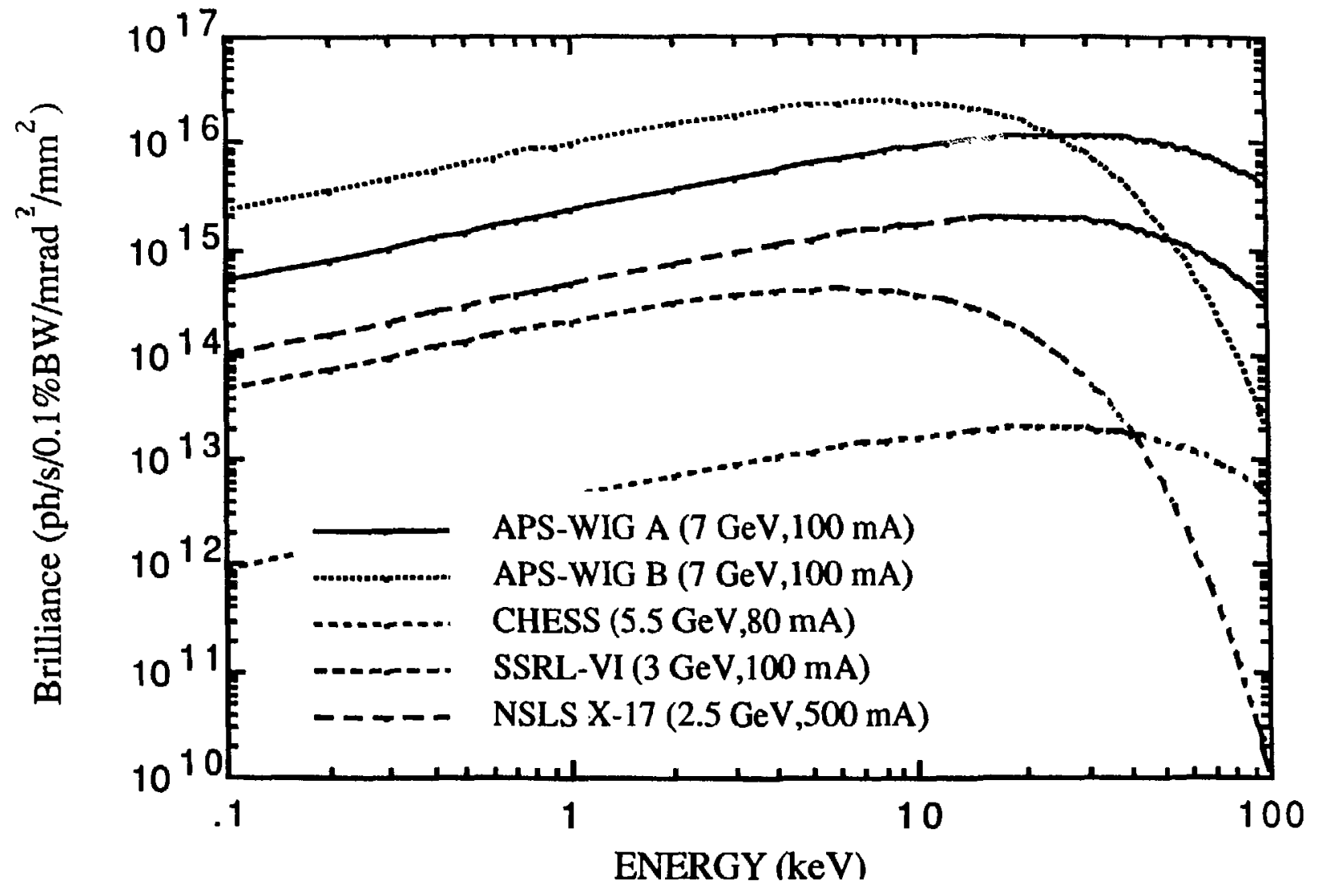

FIGURE 7.3 Central Brilliance from Wigglers on the 7-GeV APS at $100 \mathrm{~mA}$, Compared with That from Wigglers on Other Synchrotron Sources 


\section{INSERTION-DEVICE POWERS}

The total power (in watts) radiated from an insertion device of length $L$ (in meters) is given by

$$
P=0.633 E_{R}^{2} B_{o}^{2} \mathrm{I} \mathrm{L}
$$

where $E_{R}$ is the positron energy in $G e V, B_{0}$ is the peak magnetic field in $T$ experienced by the positrons, and $I$ is the stored current in $\mathrm{mA}$. The angular dependence of this power (in $W / \mathrm{mrad} \theta \cdot \mathrm{mrad} \psi$ ) is

$$
\partial^{2} P / \partial \theta \partial \psi=0.01084 E_{R}^{4} B_{0} I N G(K) f_{K}(\theta, \psi)
$$

where $\mathrm{N}$ is the number of periods in the device, and

$$
G(k)=\left(k^{7}+24 k^{5} / 7+4 k^{3}+16 k / 7\right) /\left(1+k^{2}\right)^{3.5}
$$

and $f_{k}(\theta, \psi)$ is a complex integral normalized to 1.18 For $\theta=0$ and $\psi=0$, one obtains the peak power density of this distribution, given by

$$
P_{\text {peak }}=0.01084 \quad E_{R}^{4} B_{0} I N G(K)
$$

From Eq. 13, notice that much of the variation in $G(K)$ is for $K$ values smaller than 1.0 , with $G(K=1.0)=0.94$. In the wiggler regime, $G(K)=1.0$. Hence, the variation in peak for an undulator increases as $K$ approaches 1.0 and then saturates for larger values of $K$.

The normal radiation impinging at a distance $M$ meters from the source point can be defined in terms of surface power density by

$$
W_{x y}\left(\text { watts } / \mathrm{mm}^{2}\right)=P_{\text {peak }} / M^{2}
$$

In designing optics or solving heat-flow problems, the normal surface power density provides the most important number. This power is usually spread over considerably larger surface areas by orienting the exposed surface at a small angle relative to the radiation. Often this angle is determined by the Bragg law or by critical angles of reflection for a given wavelength of radiation, depending on the optics used.

In Table 8.1, the parameters related to the power distributions from various insertion devices on the $7-\mathrm{GeV}$ APS are compared with those from a few devices on other storage rings. The total power from Undulator A will be 1.65 $\mathrm{kW}$ for $\mathrm{K}=1$, and the peak power density will be $126 \mathrm{~kW} / \mathrm{rad}^{2}$. At a distance 
of $30 \mathrm{~m}$, the normal surface power density is $140 \mathrm{~W} / \mathrm{mm}^{2}$. In the above discussion, the positrons are treated without including the spatial and angular spreads in these beams. The vertical and horizontal divergences of the source including these effects are

$$
\Sigma_{y}^{\prime}=\left[\left(\frac{0.608}{\gamma}\right)^{2}+\sigma_{y}^{21}\right]^{1 / 2}
$$

and

$$
\Sigma_{x}^{\prime}=\left[\left(\frac{K}{\gamma}\right)^{2}+\sigma_{x}^{\prime 2}\right]^{1 / 2}
$$

In the case of the APS, the radiation opening angles dominate the contribution from the particle beam divergence, and the reductions in the peak power densities associated with emittance effects are minimal. Therefore, the zero emittance values given in Table 8.1 can be considered as good estimates.

\begin{tabular}{|c|c|c|c|c|c|}
\hline Parameter & $\begin{array}{c}\text { APS } \\
\text { Undulator A }\end{array}$ & $\begin{array}{l}\text { APS } \\
\text { Wiggler } 1\end{array}$ & $\begin{array}{l}\text { APS } \\
\text { Wiggler } 2\end{array}$ & $\begin{array}{c}\text { SSRL } \\
\text { VI }\end{array}$ & $\begin{array}{l}\text { NSLS } \\
x-17\end{array}$ \\
\hline $\mathrm{E}_{\mathrm{R}}(\mathrm{GeV})$ & 7.0 & 7.0 & 7.0 & 3.0 & 2.5 \\
\hline$I(m A)$ & 100 & 100 & 100 & 100 & 500 \\
\hline$B_{0}(T)$ & $0.81^{a}$ & 1.0 & 0.3 & 1.2 & 6.0 \\
\hline$L(m)$ & 5.0 & 1.5 & 5.0 & 1.96 & 0.53 \\
\hline Periods, N & 151 & 10 & 20 & 27 & 3 \\
\hline K & 2.5 & 14 & 7 & 8 & 99 \\
\hline $\begin{array}{l}\text { Total Power } \\
(\mathrm{kW})\end{array}$ & $10 \cdot 3^{a}$ & 4.6 & 1.4 & 1.6 & 37.9 \\
\hline $\begin{array}{l}\text { Peak mrad } \\
\left(\mathrm{kW} / \mathrm{mrad}^{2}\right)\end{array}$ & $326^{a}$ & 26 & 16 & 2.8 & 3.8 \\
\hline $\begin{array}{l}W_{(W y} \\
\left(W m^{2} m^{2}\right. \\
\end{array}$ & $204^{a}$ & 16 & 10 & $28^{b}$ & $38^{b}$ \\
\hline
\end{tabular}

TABLE 9.1 Power Distributions from Insertion Devices

${ }^{a}$ Calculated for smallest gap of $1.0 \mathrm{~cm}$ and highest $\mathrm{K}$.

${ }^{b}$ Calculated at $10 \mathrm{~m}$ from the source midpoint. 


\section{SPECIAL INSERTION DEVICES}

During the next few years, many new insertion devices will be designed to tailor the radiation from the APS. These will include sources to orient the polarization axis, to produce polarized hard $x$-rays with variable ellipticity, to provide undulator radiation with broader bandwidth, to deliver two types of pseudo-monochromatic radiation, and others. In this section we briefly describe some of these special devices that have been considered for the $7-\mathrm{GeV}$ APS.

\subsection{UIDULATOR-WIGGLER}

It would be useful to have an insertion device that provided a wide variability in $K$ from 1 to 9 and could thus serve as both an undulator and a wiggler. Such a device would have many interesting applications, because it would permit different kinds of investigations to be carried ouc on a beam line without restructuring the insertion device or moving the experiment from one beam 1 ine to another. To demonstrate this capability in the case of the 7-GeV APS, a design is presented for a device with an 8-cm period.

At gaps larger than $4 \mathrm{~cm}$, the device has undulator characteristics, with $K$ varying from 1 to 2.5 . The minimum gap necessary for the device to function as a wiggler is about $1.7 \mathrm{~cm}$. With the Nd-Fe-B hybrid configuration, a $K$ value of about 9 can be achieved at this gap. The on-axis brilliance as a function of photon energy for this device is shown in Fig. 9.1 for three different values of $\mathrm{K}$. The undulator is tunable over the energy range from 1.5 to $9 \mathrm{keV}$ when the first, second, and third harmonics are used. The critical energy of the wiggler spectrum is over $30 \mathrm{keV}$.

\subsection{VERTICAL WIGGLER}

The planar wigglers commonly built provide radiation with linear polarization in the orbital plane (x-direction). There are numerous experiments that demand that the polarization axis be perpendicular to the orbital plane ( $y$-direction). This can be achieved using a wiggler with field lines in the x-direction (a "vertical" wiggler). A wiggler suitable for this purpose on the 7-GeV APS would be constructed similar to Wiggler B, with a critical energy near $10 \mathrm{keV}$.

\subsection{BROAD-BAND UNDULATOR}

While the high degree of spatial collimation of undulator radiation is generally beneficial, the very narrow energy band from various harmonics of the device is not useful for certain applications. This is especially true for spectroscopic techniques, such as EXAFS, and for techniques based on anonalous dispersion. In these cases, an energy bandwidth approximately ten times larger than that of the radiation harmonics from a transverse undulator is desirable. 


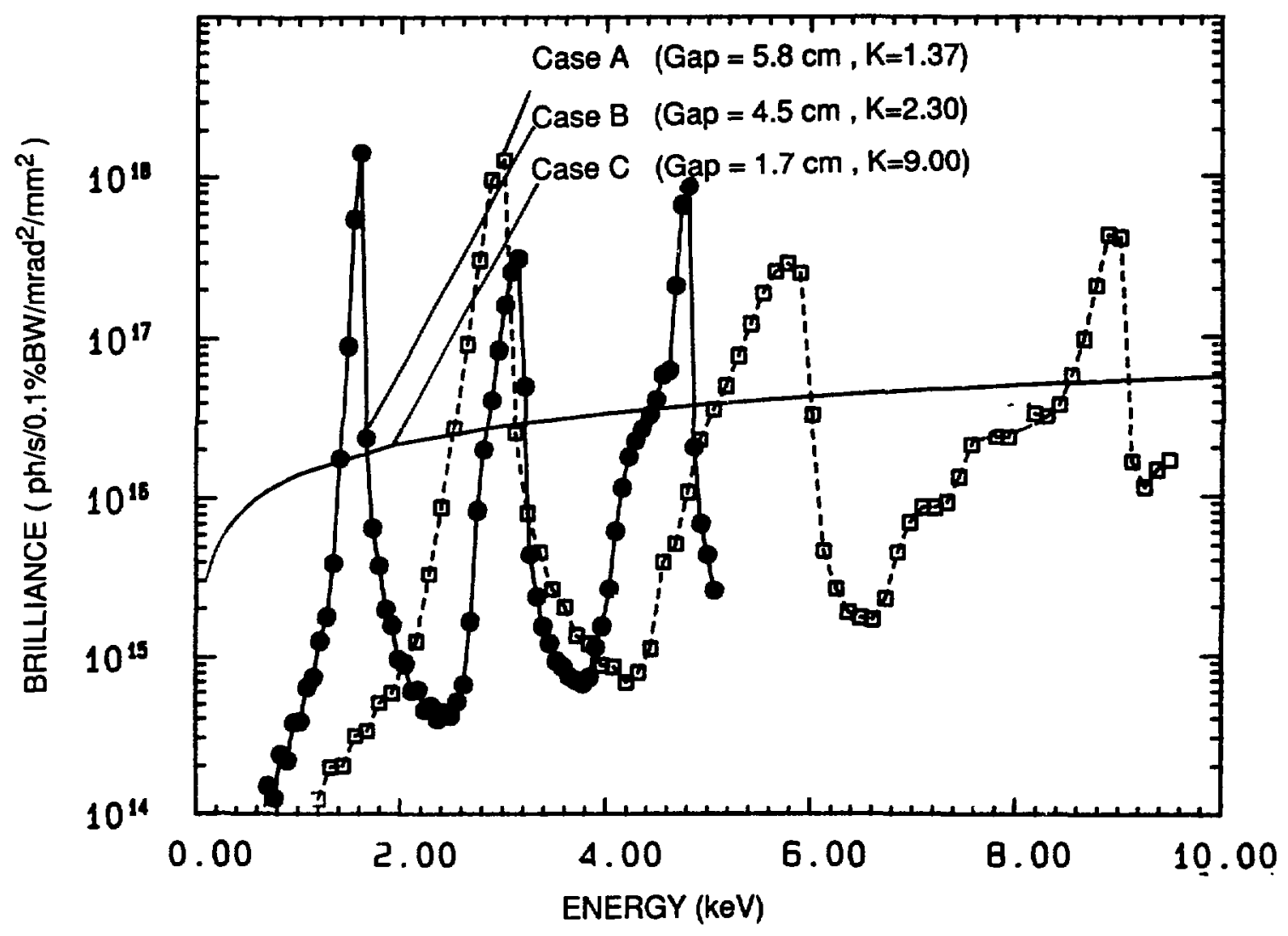

FIGURE 9.1 On-Axis Spectral Brilliance vs. Photon Energy for the UndulatorWiggler at Three Different Gaps (The R-values are also shown. The undulator spectral calculations include the phase-space dimensions of the positron beam.) 
For those who seek to use the APS for such applications, several possibilities for producing "broad energy-band" undulator radiation have been investigated. These include undulators with either a period or a magnet gap that varies linearly along its length. A varying period or gap broadens the energy-band of a given undulator harmonic, but it does not result in a $f 1$ at distribution of the photon brilliance over the energy width of the harmonic. A broad energy-band with a flat intensity distribution is obtained from an undulator by constructing an undulator with several segments. Each segment has a different gap and a different number of periods. The relative phase of the positron beam trajectory between segments is purposely not maintained through the device, and consequently the resulting photon intensity is a sum of the intensities from each segment. This has the advantage that the segments can be tailored to make this resulting intensity nearly flat over the harmonic energy bandwidth.

As an example of a broad-band segmented undulator, we have carried out simulations of the spectral characteristics of a five-segment device with a period of $3.35 \mathrm{~cm}$ and a total length of approximately $5 \mathrm{~m}$. The number of periods and the gap for each segment are adjusted so that the on-axis brilliance for the first harmonic, shown in Fig. 9.2, is nearly flat over an energy interval of about $1.2 \mathrm{keV}$ (centered at about $9.5 \mathrm{keV}$ ). The average energy position of this first-harmonic broad band can be shifted or tuned by changing the gap uniformly for all five segments. At the minimum magnet gap of $10 \mathrm{~mm}$ expected for the APS, the first harmonic is centered at approximately $5 \mathrm{keV}$ and has an energy bandwidth of about $1.8 \mathrm{keV}$. At the maximum gap, the first harmonic is centered at approximately $11.5 \mathrm{keV}$ and has an energy width of $0.7 \mathrm{keV}$. The brilliance remains flat to within a factor of two over the tunable range of the device, well within the dynamic range of most detectors.

\subsection{EXTRA-LONG STRAIGHT SECTIONS}

The straight sections on the $7-\mathrm{GeV}$ APS lattice are $6.2 \mathrm{~m}$ long and can accommodate a maximum insertion-device length of $5 \mathrm{~m}$. Thus, opportunities exist to install one long device or several shorter devices. The need for straight sections longer than $6.2 \mathrm{~m}$ was addressed by the National Task Group. This group concluded that the $6.2 \mathrm{~m}$ length is adequate for currently proposed scientific research. However, to maintain flexibility for future experiments, the APS storage-ring design is capable of accommodating extralong straight sections of $8.5-\mathrm{m}$ length. Note that the brightness of the radiation from undulators on the APS is proportional to $\mathrm{N}^{\mathrm{x}}$ with $\mathrm{x}=1.2$, rather than the $x=2$ value deduced from single-particle calculations. Hence, extra-long straight sections would not greatly enhance the brilliance through longer devices with larger number of periods. On the other hand, there may be situations where the experiment demands two or more radiation sources on a single straight section. In those cases, an extra-long straight section could accommodate many devices.

The extra-long straight section on the $7-\mathrm{GeV}$ APS storage ring is realized by the removal of a quadrupole magnet on each end of a straight section. This increases the usable length for an insertion device to about $8 \mathrm{~m}$. It is to be expected that only a few such extra-long sections will be required. 


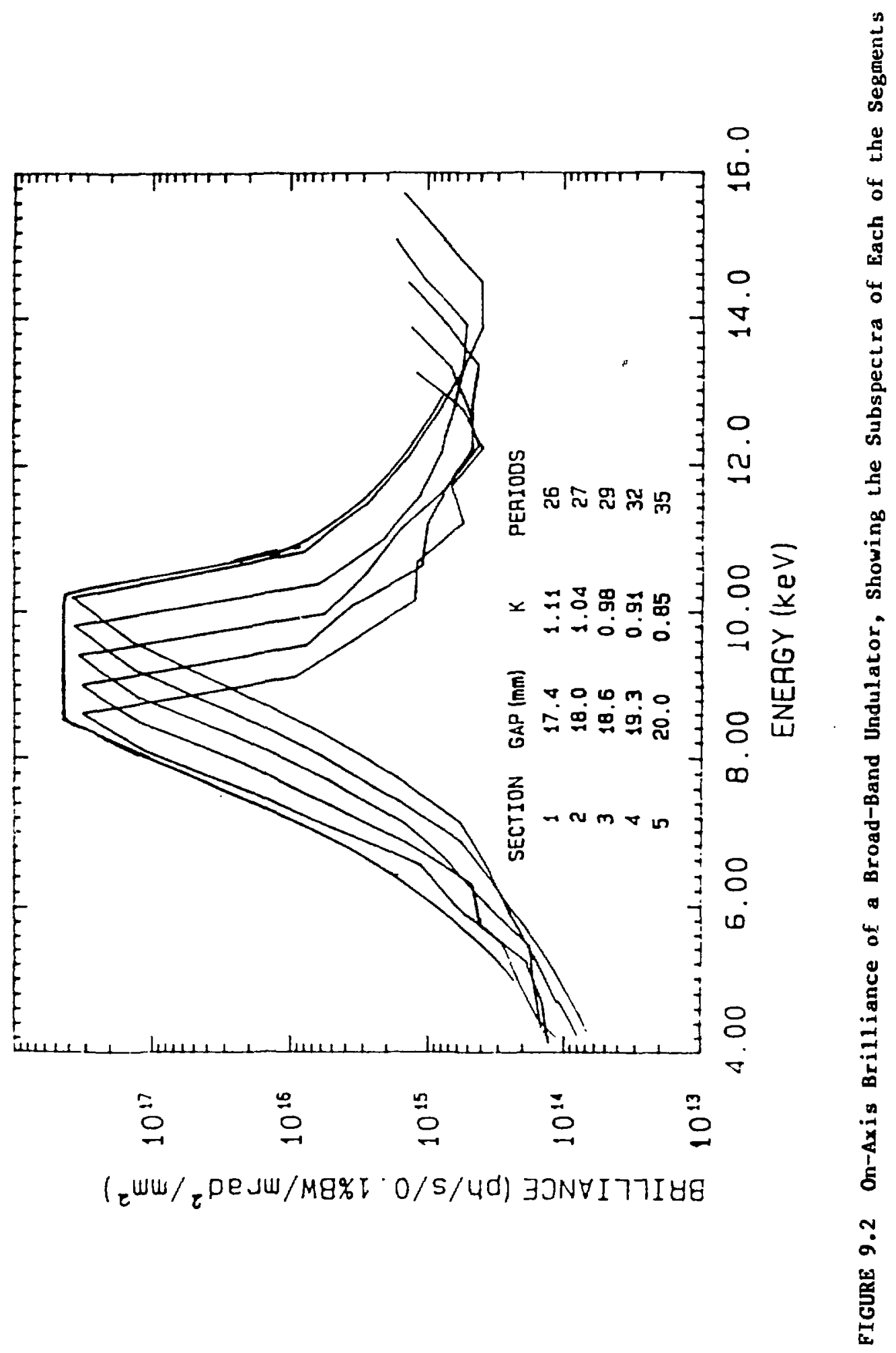




\subsection{POLARIZATIOU SOURCES}

It is anticipated that the polarization characteristics from various sources at the APS will be fully exploited.

Synchrotron radiation produced from bending-magnet sources has two polarization components; one parallel to the orbital plane, $I_{\|}$, and the other perpendicular to the plane, $I_{\text {. The }}$. Theaxis radiation is completely linearly polarized. Integrated over the vertical extents of the beam, the degree of linear polarization, $\left(I_{\|}-I_{1}\right) /\left(I_{\|}+I_{1}\right)$, is about 0.76 at the critical energy. Above and below the orbital ptane, the radiation is elliptically polarized. The degree of 1 inear polarization can be altered, albeit with a loss of flux, by accepting radiation through a vertical slit. It is estimated that a high degree of linear polarization $(0.9)$ can be obtained through this arrangement, with a modest $(<50 \%)$ sacrifice in the flux. By going above and below the orbital plane, elliptical polarization can be obtained; the helicity of the polarization is determined by the direction of the positron beam curvature relative to the observation axis.

The positron beam through a wiggler experiences oscillations in the trajectory. Hence, as in the bending-magnet source, the radiation is linearly polarized in the orbital plane. Away from this plane, we have elliptical polarizations of alternating helicity from successive poles of the wiggler, which add to destroy the net polarization, except for any residual component. The vertical wiggler discussed in Sec. 9.2 will produce polarization in the vertical ( $y z$ ) plane. An asymmetric wiggler has been proposed that would produce wiggler radiation with varying ellipticity. ${ }^{19}$

The transverse undulator radiation in the first harmonic is linearly polarized in the plane of the orbit $(x z)$. There are distinct types of undulators that can in principle deliver elliptically polarized radiation onaxis, unlike the transverse undulators of types $A, B$, and $C$ discussed earlier: They are (1) helical undulators, 20,21 (2) crossed undulators, 22 and (3) deformed helix undulators. ${ }^{23}$ More studies are needed on these devices before they can be used to produce radiation with different ellipticities on a routine basis on the APS. 


\section{TIME STRUCTURE}

\subsection{STORAGE-RING PARAMETERS}

With a radio frequency ( $R F$ ) accelerating system operating at $353 \mathrm{MHz}$ and a circumference of 1060 meters, the APS storage ring will have a harmonic number of 1248; that is, there are 1248 stable orbital positions or RF buckets, equally spaced around the ring. The explicit timing properties of the storage ring will depend on which and how many of the RF buckets are filled. Calculations indicated that the maximum achievable current per bunch is $5 \mathrm{~mA}$; hence, to attain a stored current of $100 \mathrm{~mA}$, a minimum of 20 bunches will be required. If the bunches are equally spaced, this would correspond to an interbunch period of approximately $177 \mathrm{~ns}$. The calculated low-current $(<0.5 \mathrm{~mA})$ bunch length and maximum current ( $5 \mathrm{~mA}$ ) bunch length are 38.7 ps ( $2 \times$ RMS) and 116 ps ( $2 \times$ RMS), respectively. A comparison of the temporal properties of the APS and other x-ray storage-ring sources is given in Table 10.1 .

Several questions related to the temporal properties of the APS have been asked by researchers interested in performing time-resolved $x$-ray measurements. These questions have focused on concerns about bunch size and stability and the degree of "emptiness" of RF buckets adjacent to a filled bucket. From discussions with the accelerator design group, the following estimates have been made: The number of particles in each bunch (i.e., the current per bunch) should be the same to within \pm 5\% at the start of a fill. A conservative estimate of the "time jitter" between the arrival of consecutive bunches should be less than $1 \sigma$ of the bunch length. This corresponds to \pm 58 ps in $177 \mathrm{~ns}$ ( 20 bunches equally distributed around the ring) or $\mathbb{1}_{0.03 \%}$ of the interpulse period. The accelerator physicists were in general agreement that the buckets adjacent to filled buckets would in fact be quite empty, in part because the injected bunch length is $1 \mathrm{~ns}$ while the RF bucket is $2.83 \mathrm{~ns}$ in length.

\subsection{SPECIAL TIMING OPTIONS}

Some portion of the user community has expressed interest in the possibility of having a single pulse of $x$-rays surrounded by "long" dark periods. ("Long" periods might range from microseconds to seconds, depending on the particular user.) One way to accomplish this is through the use of fast kicker magnets that could steer a positron bunch (or several bunches) out of the normal orbit for a short duration and then deflect it back. The timing experiment would be arranged so that it would receive radiation only from the deflected bunch(es). However, there is considerable concern that these wobbler magnets could have deleterious effects on the spatial and angular stability of the positron beam in general.

We have recently been exploring an alternative approach to this problem, one utilizing asymmetrically spaced bunches in the storage ring coupled with mechanical choppers. One proposal calls for placing 19 of the 20 circulating bunches in contiguous $\mathrm{RF}$ buckets and the remaining bunch diametrically opposite to them. This particular arrangement would allow the use of a mechanical chopper, rotating at 18,000 RPM, to pick out and transmit radiation from the lone pulse, so that an interpulse period of 3.536 us (the natural 
Table 10.1 Time Structures of Various storage Rings

\begin{tabular}{lllll}
\hline \multicolumn{1}{c}{ Parameter } & SSRL & CHESS & NSLS & APS \\
\hline & & & & \\
Orbit Period (ns) & 760 & 2560 & 568 & 3536 \\
Number of Bunches & $1(4)$ & 7 & 30 & $1(20)$ \\
Bunch Duration (ps) & 300 & 160 & 1700 & 116 \\
$\begin{array}{l}\text { Interpulse Period a } \\
\text { (ns) }\end{array}$ & $760(190)$ & 366 & 18.9 & $3536(177)$ \\
\hline
\end{tabular}

${ }^{a}$ Numbers in parentheses refer to alternative modes of operation.

orbital period) could be achieved. With a single chopper, tailoring the slot configuration could allow any dark time from 3.536 us to $3.33 \mathrm{~ms}$ (corresponding to a single slotced chopper wheel). For longer interpulse periods, two choppers in series can be used. In this configuration, the second chopper rotates at a slightly different frequency, resulting in alignment of the two slots at times given by the beat frequency between the two wheels. The twowheel scenario has the additional advantage that by counter-rotating the wheels, only half the rotational frequency per chopper wheel is required. With a $\Delta v$ of 20 RPM (the minimum allowable value so that there is no slot overlap at the arrival of a burst of $x$-rays immediately following the one that was just passed), a 6-s interpulse period can be realized. An effort to investigate the mechanics and electronics of such chopper-wheel arrangements is under way.

\subsection{TIMING DETECTORS}

Although the temporal properties of the storage ring have been emphasized, clearly many experiments will not need to explicitly utilize the modulated time structure of the storage ring to perform time-resolved experiments, but will instead rely on special detectors. The required characteristics of the detectors are experiment-specific. However, such properties as high data-rate capabilities, fast readout, large dynamic ranges, time-slicing capability, good spatial resolution, and gatability are all desirable, in some combination or another; much work in the area of detector development will be required to fully utilize the enhanced brightness that the APS will afford over currently operating sources. One such detector development program is currently under way in a collaboration between ANL and the University of Michigan. 


\subsection{FLUX PER BUNCH FOR VARIOUS APS SOURCES}

Table 10.2 lists the calculated flux per bunch for both bending-magnet and insertion-device sources at the APS. A11 calculations are made at the critical energy (for bending-magnet and wiggler sources) or at the fundamental energy (for undulator sources), assuming 7.0-Gev, 100-mA operation with 20 bunches.

By adjusting the bandwidth of the monochromating system, large increases in flux per pulse can be obtained with bending-magnet and wiggler sources and (to a lesser extent) with undulator sources, since these have a natural line width of 1 to $5 \%$.

Table 10.2 Flux per Bunch for APS X-Ray Sources

\begin{tabular}{|c|c|c|}
\hline Source & Flux & \\
\hline Bending Magnet & $1.7 \times 10^{6} \times$-rays $/ 0.1 \%$ & $\mathrm{BW} \cdot \mathrm{mrad} \theta$ \\
\hline Wiggler A & $3.9 \times 10^{7} \times$-rays $/ 0.17$ & $\mathrm{BW} \cdot \operatorname{mrad} \theta$ \\
\hline Wiggler B & $4.5 \times 10^{7} \times$-rays $/ 0.17$ & $\mathrm{BW} \cdot \mathrm{mrad} \theta$ \\
\hline Undulator $A$ & $2.1 \times 10^{8} \times$-rays $/ 0.17$ & $\mathrm{BW}$ \\
\hline Undulator B & $1.7 \times 10^{8} \mathrm{x}$-rays $/ 0.17$ & $\mathrm{BW}$ \\
\hline
\end{tabular}




\section{EXPERIMENTAL FLOOR AND LABORATORY OFFICE HODULES}

\subsection{GEMERAL LAYOUT}

The 7-GeV APS design provides for supporting a large number of beam lines and experimental stations to meet the demands of a wide variety of users. The storage ring will include up to 35 ports delivering bending-magnet radiation and 34 straight-section ports delivering insertion-device radiation.

One of the key issues in beam-line design involves the ability to handle unprecedented power densities and power gradients from the insertion devices. Considerable R\&D work is being undertaken to develop the design for the first optical element; this effort borrows from the advanced methods and technologies used in other areas, such as fission and fusion. The cooling of the optical element using liquid metals is a part of this R\&D work.

A closely related issue concerns the length of the beam lines. Provision has been made to accommodate $80-m-10 n g$ beam lines within the experimental hall. This length is governed by several factors, the primary one being the location of the first optics. Finite-element analysis of a Si premonochromator cooled with liquid Ga shows that, with careful engineering, it can be located 30 to $60 \mathrm{~m}$ from the source (depending on the insertion device) without undergoing unacceptably large distortions.

Some applications will demand much longer beam 1 ines, extending out of the experimental hall. In the present placement of laboratory/office modules around the ring, there are at least six locations where the beam 1 ines could extend beyond the experimental hall without any building obstruction.

A tentative plan for disposition of the beam 1 ines is shown in Fig. 11.1 in order to suggest the magnitude of this facility. Enough flexibility is built into the present design to accommodate anticipated changes in device distribution, as well as experimental stations. The capability to install large experimental stations and accommodate additional user needs on the experimental floor is a principal feature of the present design.

\subsection{LABORAIORY/OFFICE MODULES FOR USERS}

At present, eight laboratory/office-module buildings can be constructed around the perimeter wall of the expeximental hall. The modules provide office and laboratory space for researchers working with beam lines in the experimental hall.

Figure 11.2 depicts a typical laboratory/office module. The design provides convenient access to laboratory, office, service and delivery, and related support spaces to maximize the experimenters' use of the facility. Each sector of the storage ring supports an insertion-device beam line and a bending-magnet beam line. Every sector has easy access to two standard laboratories, eight offices, and additional space for a small meeting room or secretarial area. Thus, each of the laboratory/office modules provides space for users working on eight beam lines contained in four sectors, as shown in Fig. 11.2. The module, in addition, has truck delivery access, vending space, 


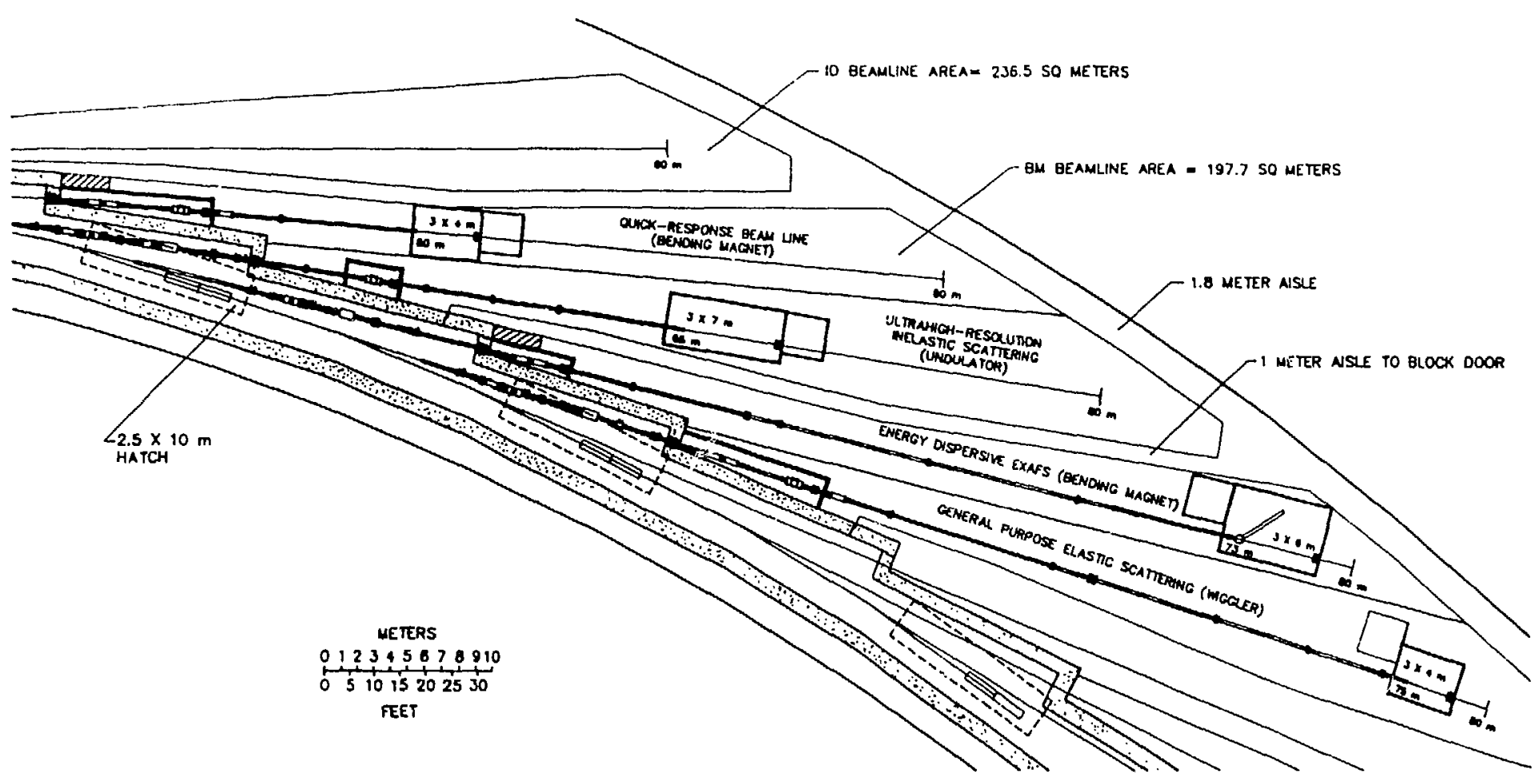

FIGURE 11.1 Layout of Typical Beam Lines in a Part of the SLorage-Ring Experimental Hall (These beam lines represent use of undulator, wiggler, and bending magnet sources.) 


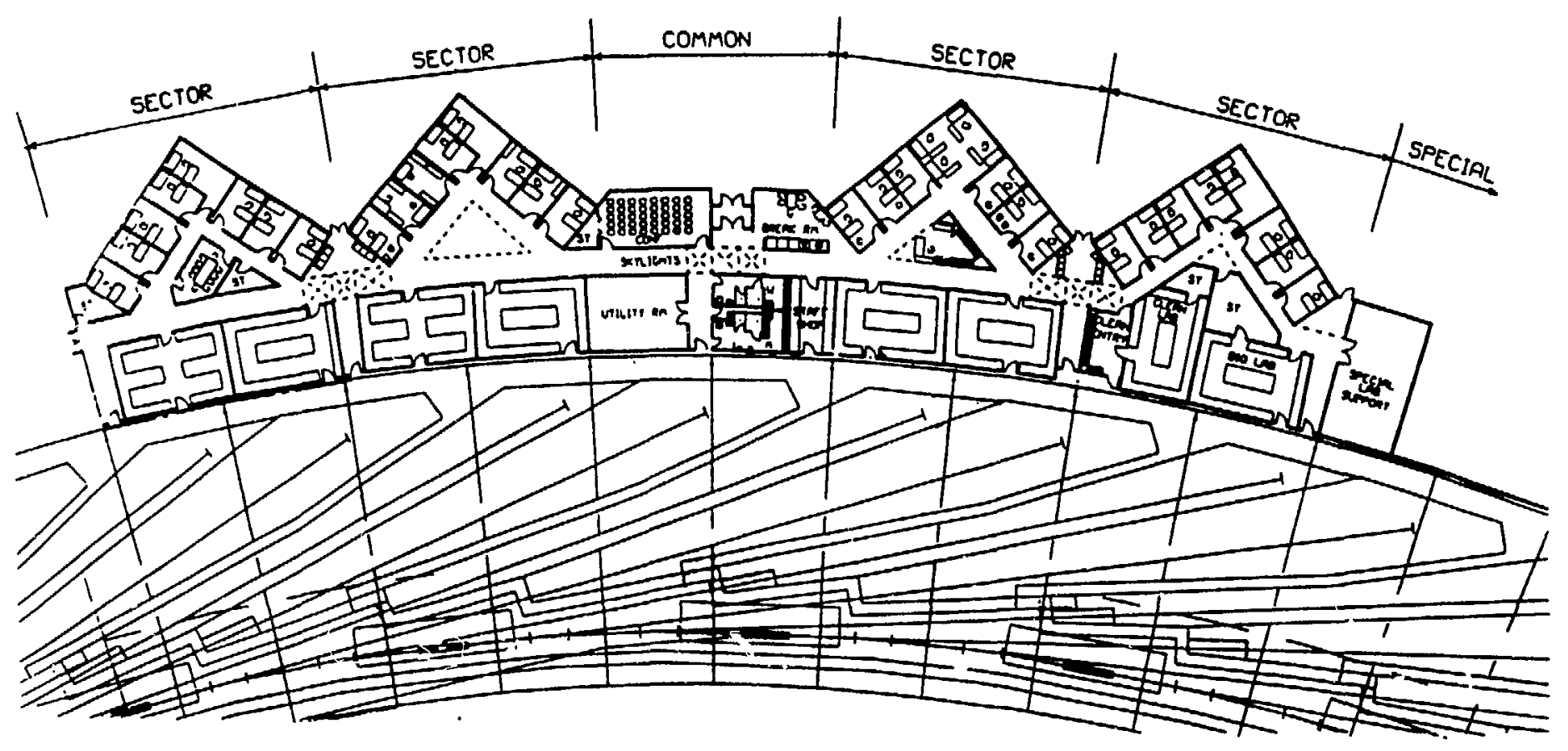

FICURE 11.2 Typical Laboratory/Office Module Providing Support for Four Insertion Devices and Four Bending-Magnet Beam Lines (Eight such modules surround the perimeter of the storage ring to provide laboratory/office needs to users at 64 beam lines.) 
locker rooms, and a small staff shop. Eight such modules will support users at 32 sectors or 64 beam lines. 


\section{REFERENCES}

1. G. Brown, Y. Cho, J. Hastings, S. Krinsky, D. Moncton, G.K. Shenoy (Chair), and P.J. Viccaro, "Ring Energy Selection and Extra-Long Straight Sections for the Advanced Photon Source: A Report and Recommendations by the National Task Group," Argonne National Laboratory Report ANL-87-14 (April 1987).

2. "Report of Machine Workshop on the 6-GeV Synchrotron Radiation Source," National Bureau of Standards, Gaithersburg, Md. (March 1985).

3. RF Design Workshop for the 7-GeV Advanced Photon Source, Argonne National Laboratory, Argonne, Ill. (Nov. 1987).

4. Report of the Energy Research Review of the 7-GeV Advanced Photon Source, E. Temple (Chair), Washington, D.C. (1987).

5. "Beport on the Workshop on the Scientific Case for a 6-GeV Synchrotron Source," Argonne National Laboratory, Argonne, Ill., Dec. 9-11, 1985, U.S. Department of Energy, Office of Basic Energy Sciences, Washington, D.C. (1986).

6. "X-Ray Synchrotrons and the Development of New Materials: Workshop Report," S. Durbin and D. Cox (Co-Chairs), Argonne National Laboratory Report ANL/APS-TM-1 (Feb. 1988).

7. "X-Ray Synchrotrons and New Opportunities in the Earth Sciences: Workshop Report," J. V. Smith and M. Manghnani (Co-Chairs), Argonne National Laboratory Report ANL/APS-TM-3 (March 1988).

8. "Time-Resolved Studies and Ultrafast Detectors: Workshop Report," R. Clarke, P. Sigler, and D. Mills (Co-Chairs), Argonne National Laboratory Report ANL/APS-TM-2 (Feb. 1988).

9. "Proceedings of the First Users Meeting for the Advanced Photon Source," Argonne National Laboratory, Argonne, I11., Nov. 13-14, 1986, Argonne National Laboratory Report ANL/APS-CP-1 (Feb. 1988).

10. G.K. Shenoy and P.J. Viccaro, "An Overview of the Characteristics of the 6-GeV Synchrotron Radiation: A Preliminary Guide for Users," Argonne National Laboratory Report ANL-85-69 (Oct. 1985).

11. "Characteristics of the Insertion Devices for the 7-GeV Synchrotron Source," Supplement A, Conceptual Design Report, Argonne National Laboratory, March 1986, Light Source Report LS-52.

12. "7-GeV Advanced Photon Source: Conceptual Design Report," Argonne National Laboratory Re רort ANL-87-15 (April 1987).

13. "Report of a Program Review: Synchrotron Radiation Source Research and Development," Ames Laboratory, Iowa State University, Ames, Iowa (Oct. 1984). 
14. "6-GeV Synchrotron X-Ray Source Conceptual Design Report," Argonne National Laboratory Report ANL-86-6 (Feb. 1986).

15. S. Bartalucci, European Synchrotron Radiation Project Report ESRP-IRM68/84; A. Luccio, European Synchrotron Radiation Project Report ESRP-IRM$12 / 84$.

16. G.K. Shenoy, P.J. Viccaro, and S. Kim, Argonne National Laboratory, unpublished information (1986).

17. G.K. Shenoy and P.J. Viccaro, A.rgonne National Laboratory, unpublished information (April 1987).

18. K.-J. Kim, Nuclear Instruments and Methods A246:67 (1986).

19. J. Goulon, P. Elleaume, and D. Raoux, Nuclear Instruments and Methods A254:71 (1987).

20. B. M. Kincaid, Journal of Applied Physics 48:2684 (1977).

21. K. Halbach, Nuclear Instruments and Methods 187:109 (1981).

22. K.-J. Kim, Nuclear Instruments and Methods 222:11 (1984).

23. S. Yamamoto and H. Kitamura, Japanese Journal of Applied Physics 26:L1613 (1987). 
Distribution for ANL-88-9

Internal:

F.C. Bennett

M.B. Brodsky

$Y$. Cho

F.Y. Fradin

R.H. Huebner
D.M. Mills

D.E. Moncton

G.K. Shenoy (425)

L. Stock

P.J. Viccaro
ANL Patent Dept. ANL Contract File ANL Libraries TIS Files (3)

\section{External :}

DOE-TIC, for distribution per UC-414 (40)

DOE Chicago Operations office:

Manager

D.L. Bray

F. Herbaty

V.H. Humme1

Advanced Photon Source Users Organization Steering Committee:

B. Batterman, Cornell University

A. Bienenstock, Stanford Synchrotron Radiation Laboratory

R.W. Broach, Allied-Signal

K. Cantwell, Stanford Synchrotron Radiation Laboratory

R. Clarke, University of Michigan, Ann Arbor

J.B. Cohen, Northwestern University

P. Eisenberger, Exxon Research and Engineering

K. Hodgson, Stanford University

P.M. Horn, IBM, T.J. Wat son Laboratory

M. Knotek, National Synchrotron Light Source, Brookhaven National Laboratory

D.W. Lynch, Ames Laboratory, Iowa State University

D.B. McWhan, AT\&T Bell Laboratories/Brookhaven National Laboratory

K. Moffat, Cornell University

R. Siemann, Cornell University

W.J. Trela, Los Alamos National Laboratory

J.H. Weaver, University of Minnesota 